\title{
Reduced reaction model for secondary gas phase in biomass gasification
}

\author{
António R. Martins, Ana Isabel Ferreiro*, Raquel Segurado, Miguel A.A. Mendes \\ IDMEC, Instituto Superior Técnico, Universidade de Lisboa, Av. Rovisco Pais, 1049-001, Lisboa, \\ Portugal \\ *Corresponding author: ana.ferreiro@tecnico.ulisboa.pt
}

\section{Supplementary information}

Figures S1 to S4 show the data of the reduced mechanisms obtained in the species and reactions reduction steps for the different operating conditions selected that undergo the reduction process: a) Mean error for mechanisms originated from species reduction, b) Contribution of the peaks and yields errors to the mean error (\%) and c) Mean error for mechanisms originated from species and reactions reduction. The similarity between the variation of the percentage of elimination reactions (black line) for different species error margins is also verified for the five different operating conditions, so for the operating conditions present in the supplementary information, the eliminated reactions percentage is not shown. 


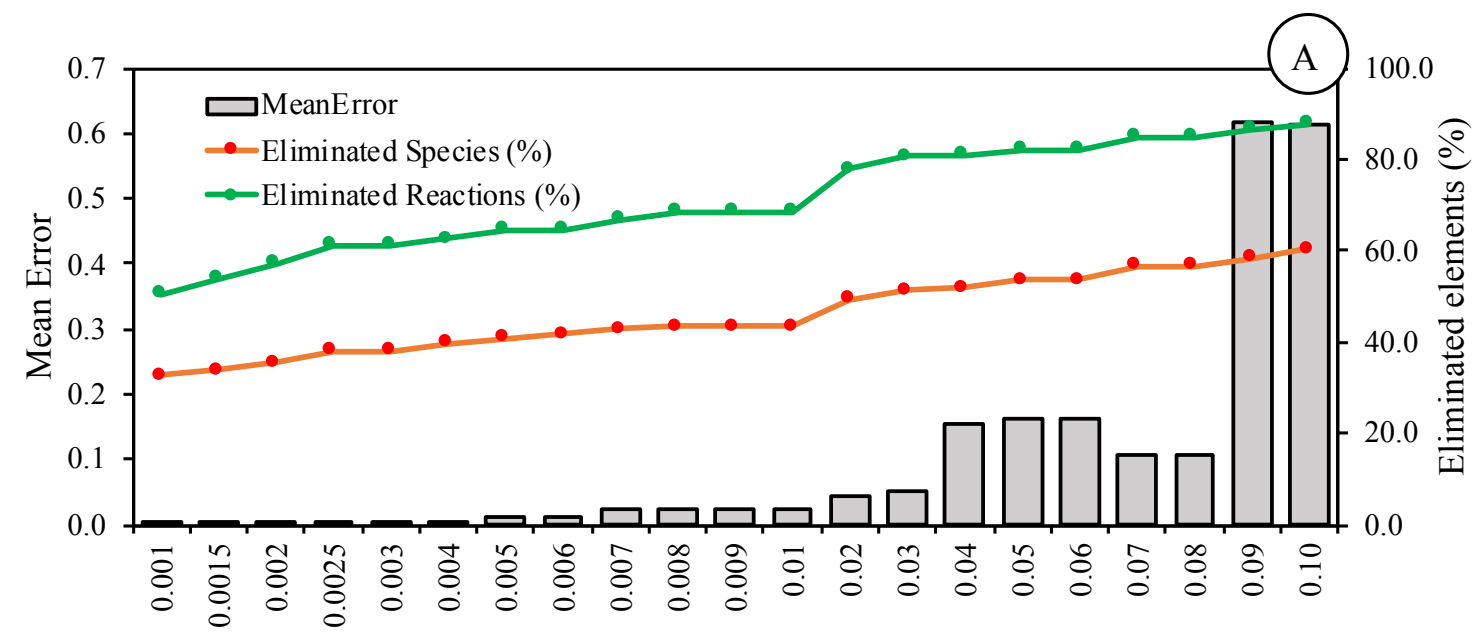

Species Error Margin

B

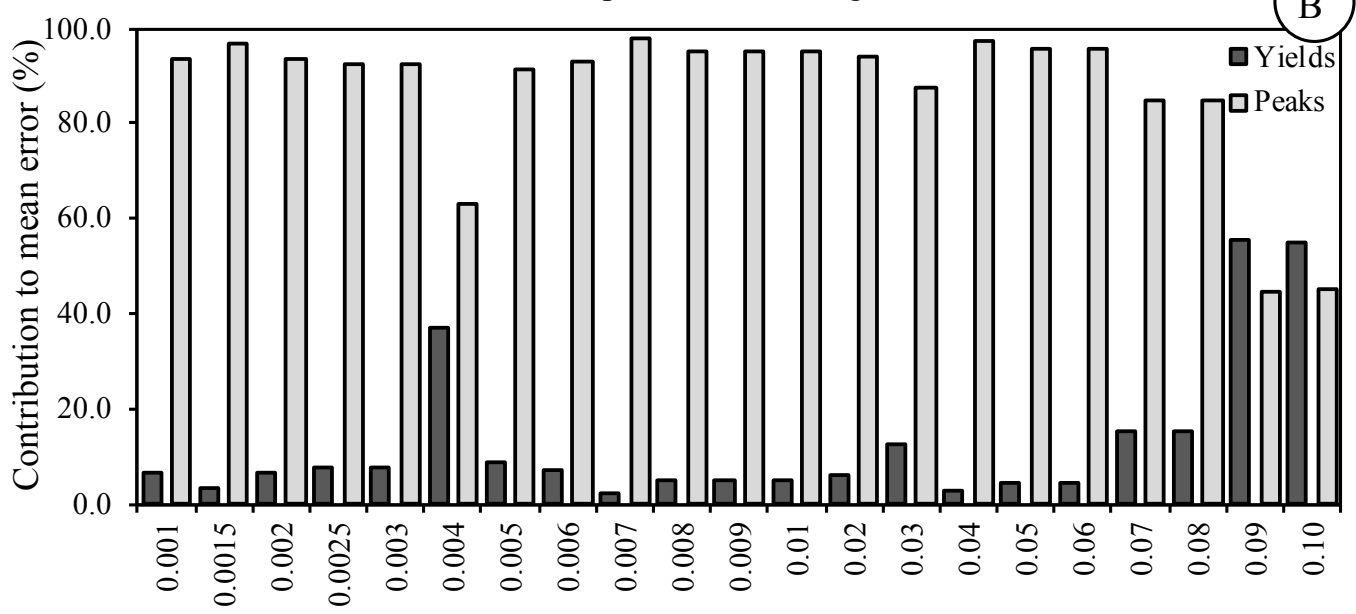

Species Error Margin

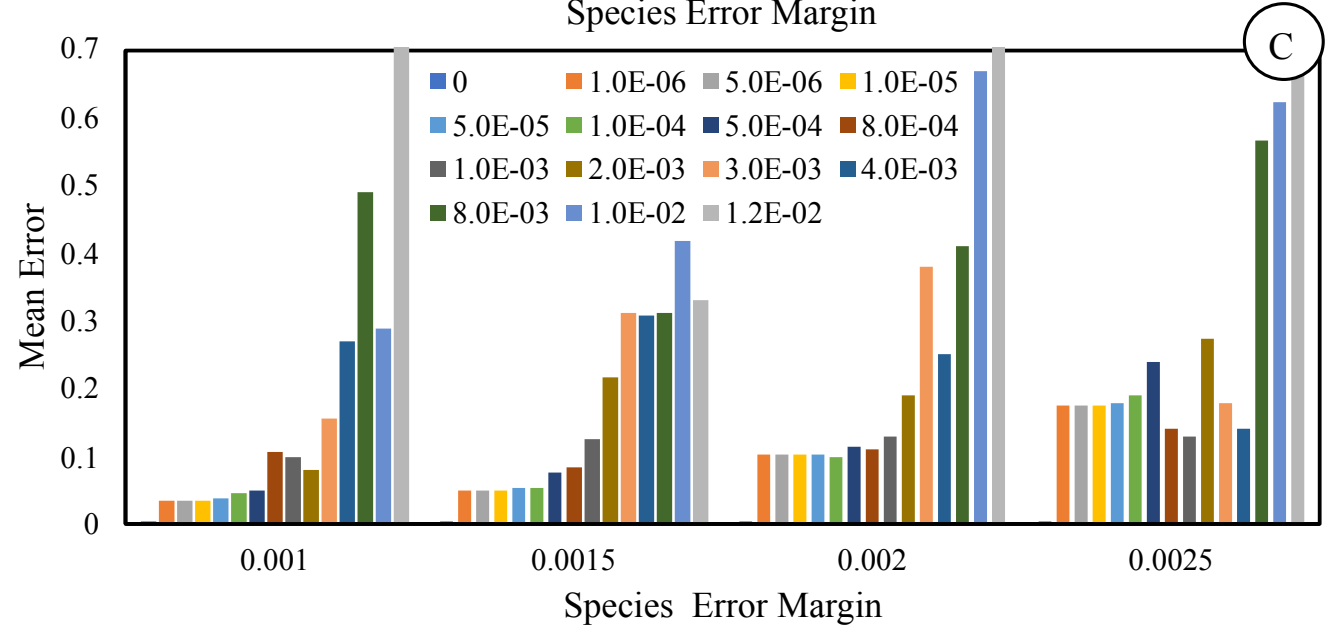

Figure S1. Temperature $=900{ }^{\circ} \mathrm{C}$, Steam to biomass ratio $=0$ 


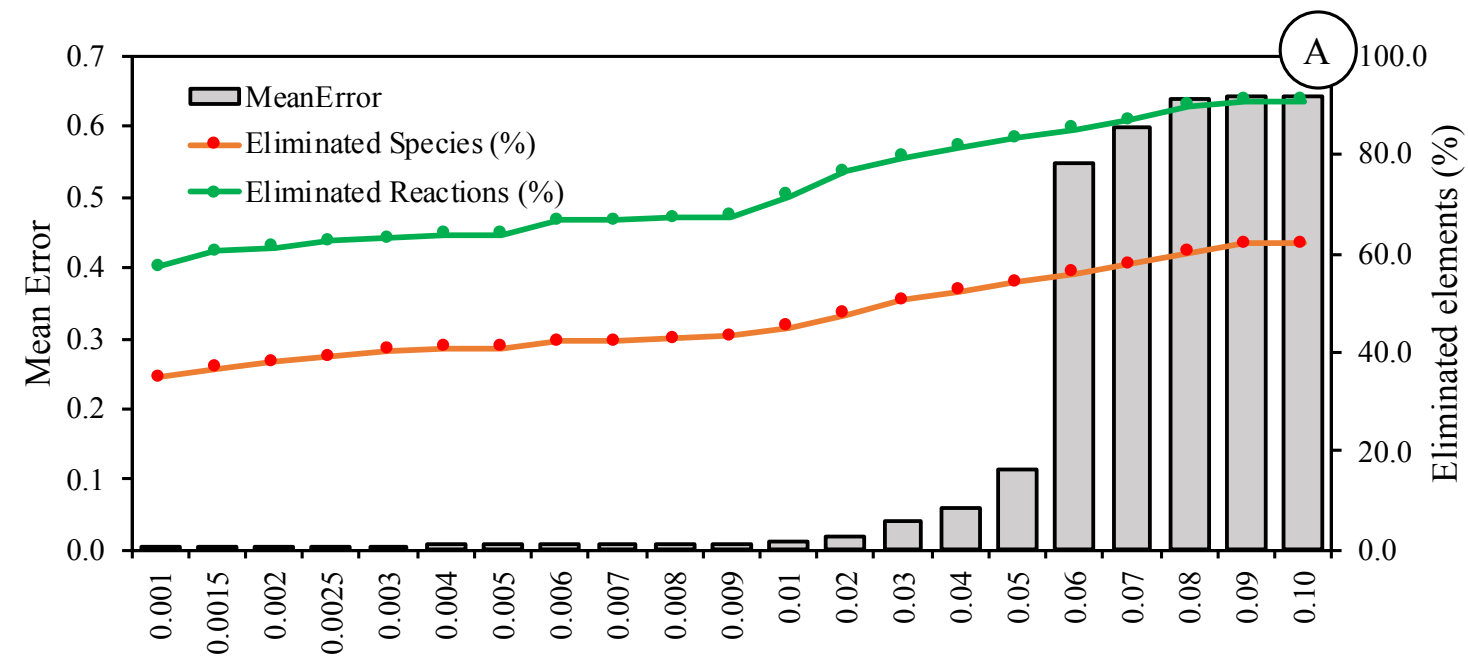

Species Error Margin
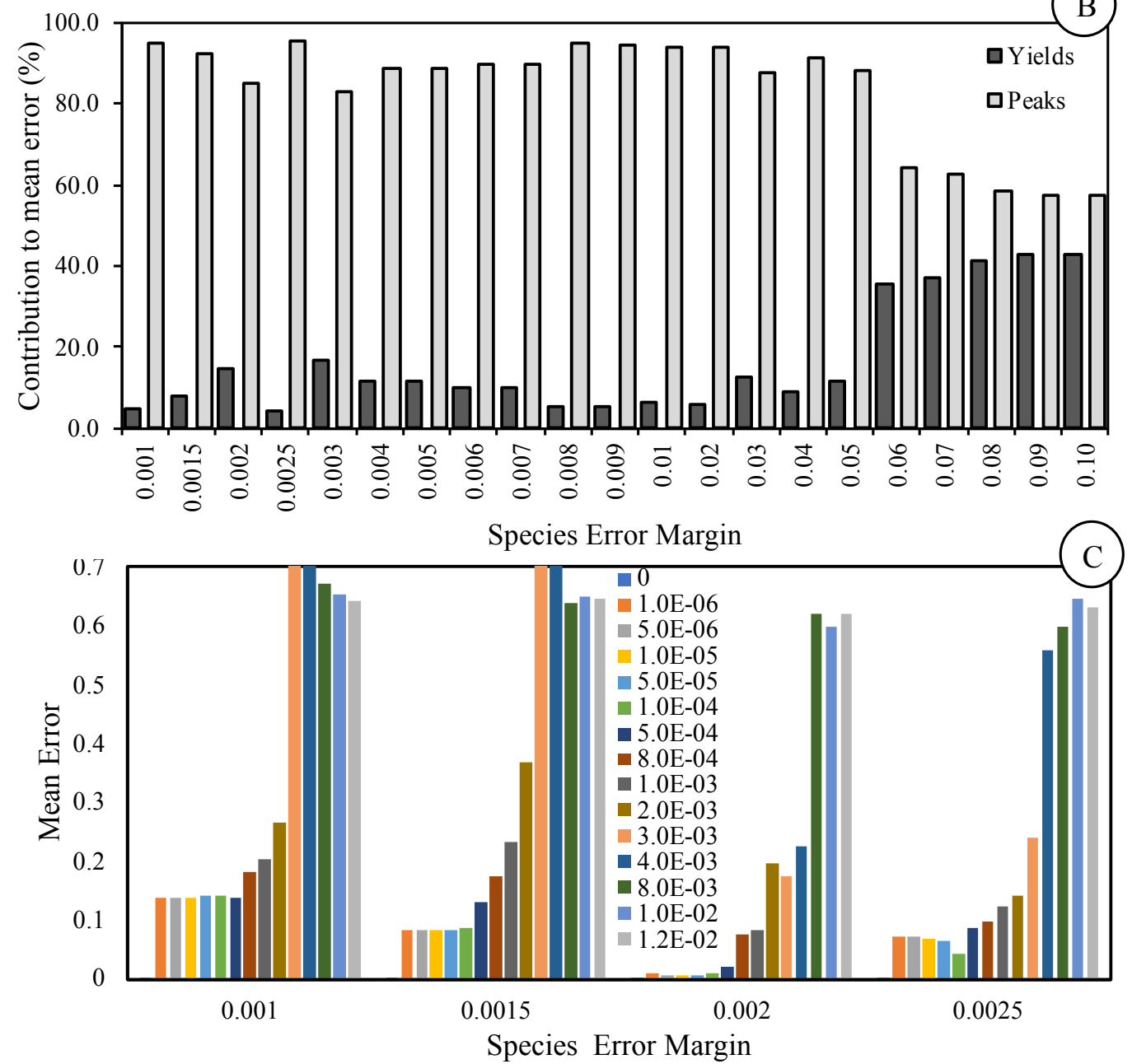

Figure S2. Temperature $=900{ }^{\circ} \mathrm{C}$, Steam to biomass ratio $=1.7$ 


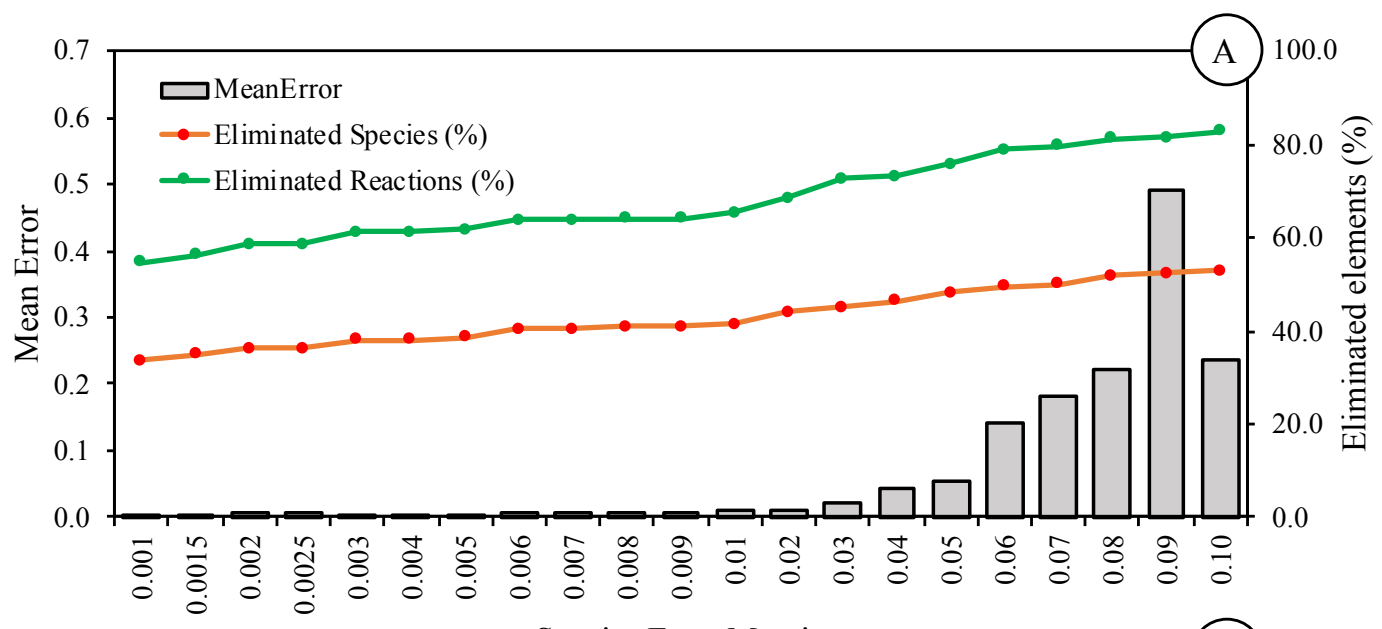

Species Error Margin

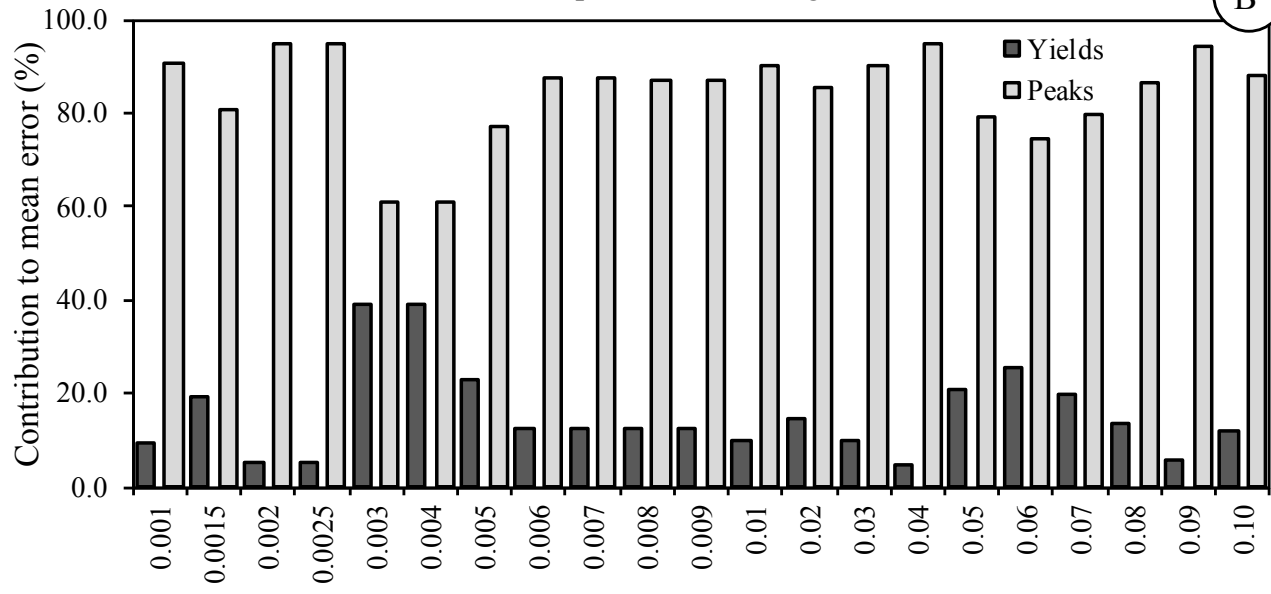

Species Error Margin

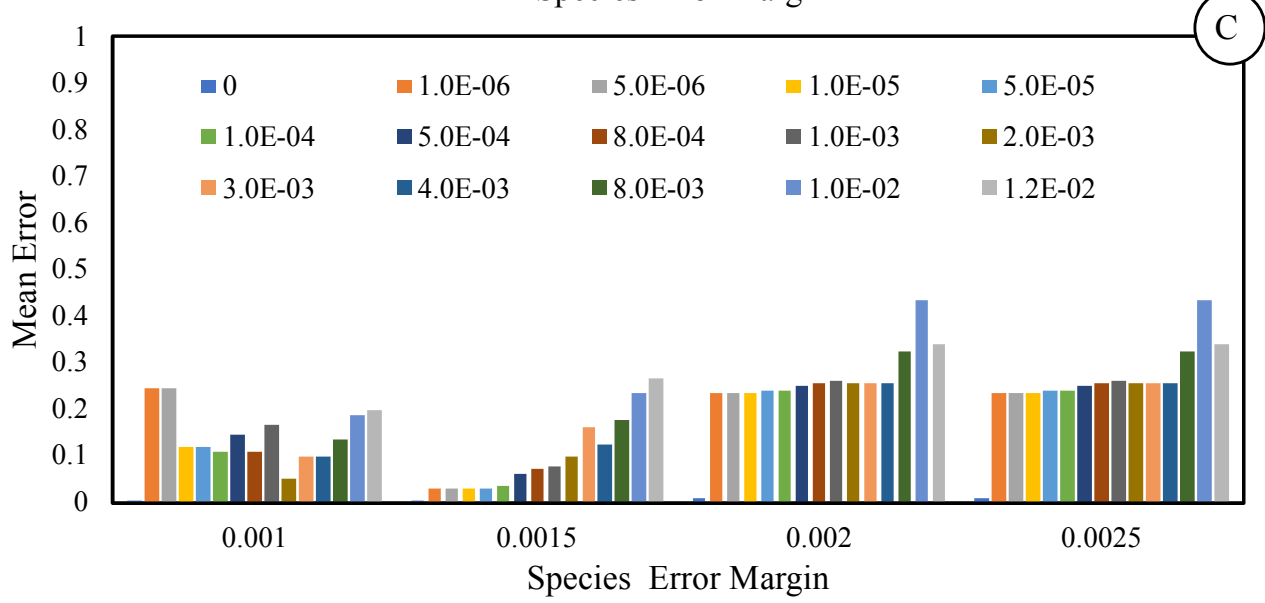

Figure S3. Temperature $=1050{ }^{\circ} \mathrm{C}$, Steam to biomass ratio $=0.85$ 


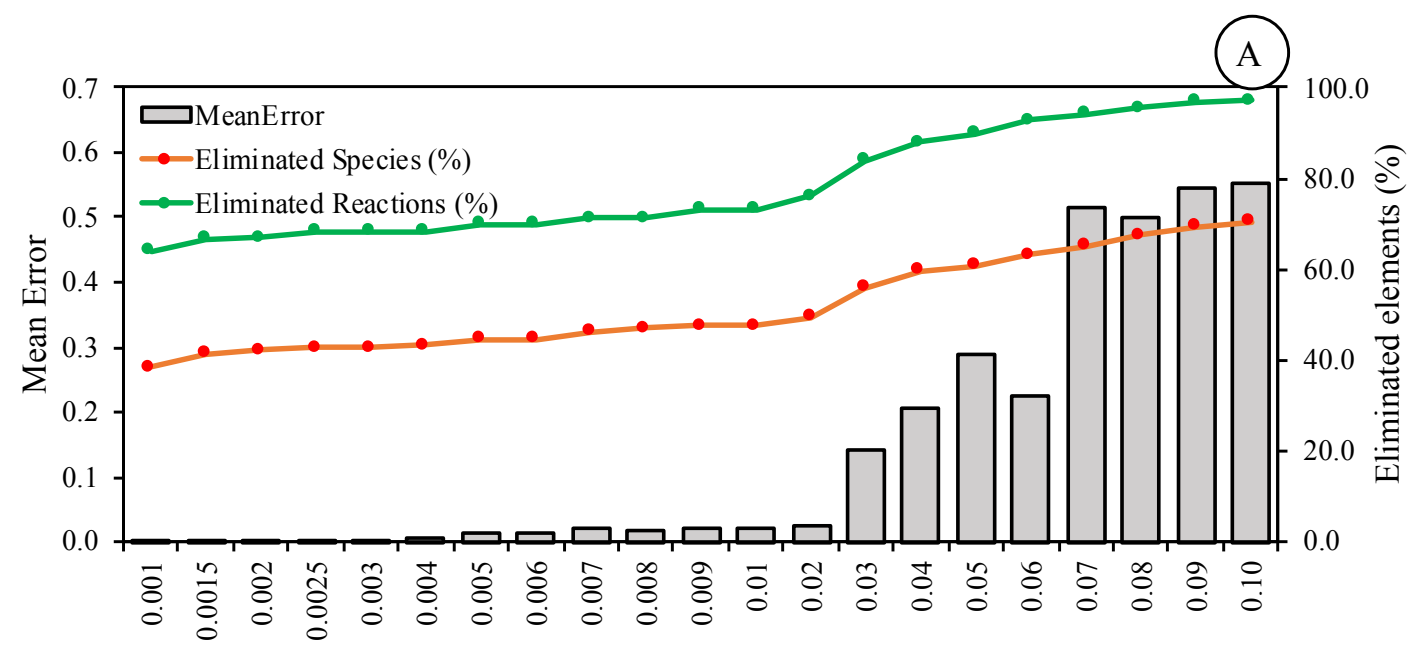

Species Error Margin
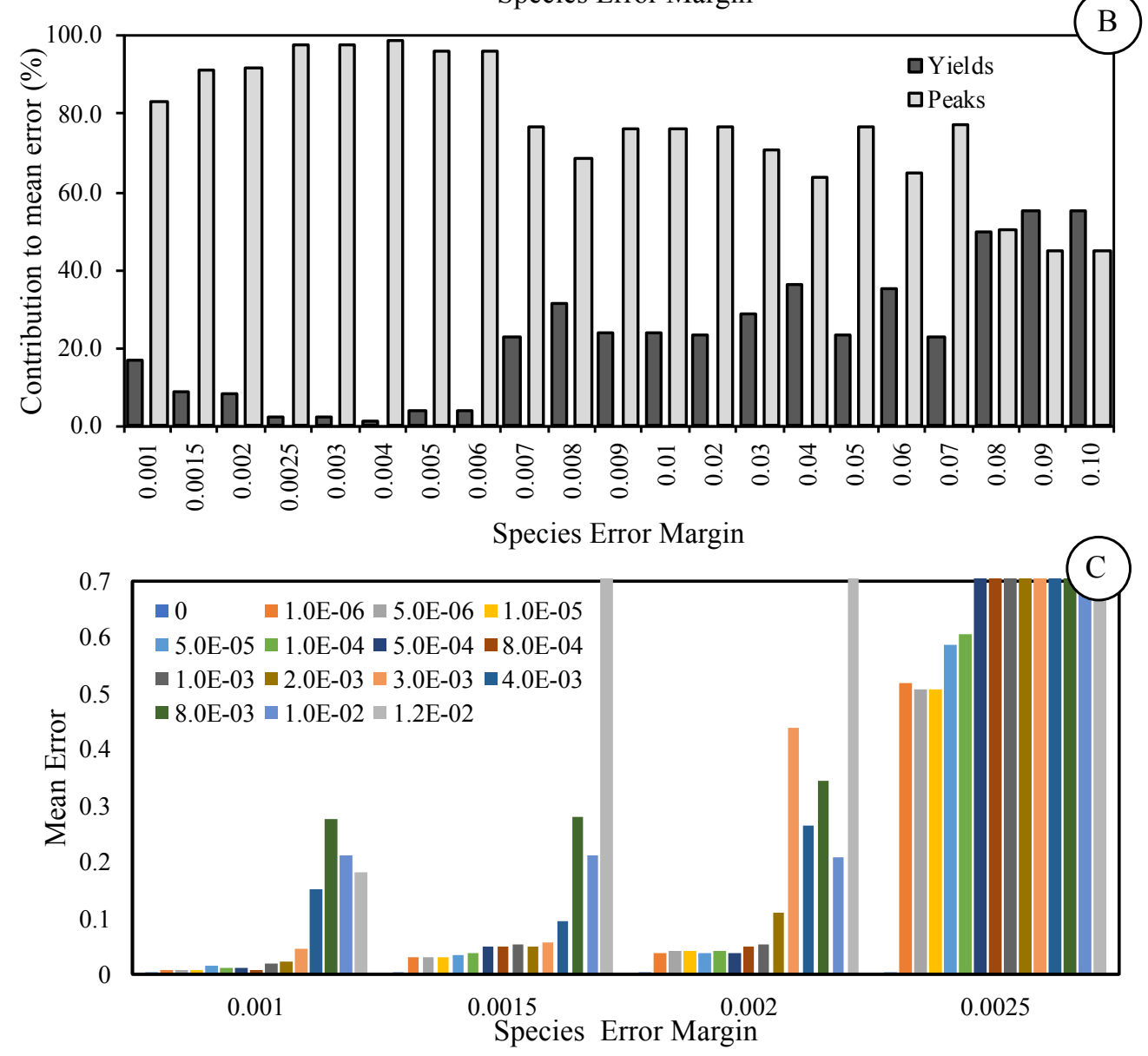

Figure S4. Temperature $=1200{ }^{\circ} \mathrm{C}$, Steam to biomass ratio $=1.7$

The reactions of reduced mechanism $\mathbf{J}$, along with the three parameters of the Arrhenius equation with the first number being the pre-exponential factor $\left(\mathrm{s}^{-1}\right)$, the second the temperature exponent and the third the activation energy (cal/mol) are presented below: 
reaction('H + O2 $\left.<=>\mathrm{O}+\mathrm{OH}^{\prime},[9.600000 \mathrm{e}+14,-0.2,16625.0]\right)$

reaction(' $\left.\mathrm{H} 2+\mathrm{O}<=>\mathrm{H}+\mathrm{OH}^{\prime},[4.330000 \mathrm{e}+13,0.0,10000.0]\right)$

falloff_reaction $(' \mathrm{H}+\mathrm{O} 2(+\mathrm{M})<=>\mathrm{HO} 2(+\mathrm{M})$ ',

$\mathrm{kf}=[5.580000 \mathrm{e}+12,0.4,0.0]$,

$\mathrm{kf} 0=[8.400000 \mathrm{e}+17,-0.8,0.0]$,

efficiencies='AR:0.8 CO:1.2 CO2:2.4 H2:2.5 H2O:18.0 HE:0.8 N2:1.26 ',

falloff $=\operatorname{Troe}(\mathrm{A}=0.5, \mathrm{~T} 3=1 \mathrm{e}-30, \mathrm{~T} 1=1.0000000000000002 \mathrm{e}+30, \mathrm{~T} 2=0.0))$

reaction $(' \mathrm{HO} 2+\mathrm{OH}<=>\mathrm{H} 2 \mathrm{O}+\mathrm{O} 2$ ', [5.000000e+13, 0.0, 1000.0])

reaction('H + HO2 <=> 2 OH', [2.500000e+14, 0.0, 1900.0])

reaction('HO2 $\left.+\mathrm{O}<=>\mathrm{O} 2+\mathrm{OH}^{\prime},[3.250000 \mathrm{e}+13,0.0,0.0]\right)$

reaction('2 $\left.\mathrm{OH}<=>\mathrm{H} 2 \mathrm{O}+\mathrm{O}^{\prime},[3.570000 \mathrm{e}+04,2.4,-2110.0]\right)$

reaction $(' \mathrm{H}+\mathrm{HO} 2<=>\mathrm{H} 2+\mathrm{O} 2$ ', [2.500000e+13, 0.0, 700.0])

reaction('2 HO2 <=> H2O2 + O2', [2.110000e+12, 0.0, 0.0])

falloff_reaction('2 $\mathrm{OH}(+\mathrm{M})<=>\mathrm{H} 2 \mathrm{O} 2(+\mathrm{M})$ ',

$\mathrm{kf}=[7.400000 \mathrm{e}+13,-0.37,0.0]$,

$\mathrm{kf0}=[1.300000 \mathrm{e}+18,-0.9,-1700.0]$,

efficiencies='AR:0.7 C2H6:3.0 CH4:2.0 CO:1.5 CO2:2.0 H2:2.0 H2O:6.0 HE:0.7 N2:0.9 ',

falloff $=\operatorname{Troe}(\mathrm{A}=0.7346, \mathrm{~T} 3=94.0, \mathrm{~T} 1=1756.0, \mathrm{~T} 2=5182.0))$

reaction('HCO + $\mathrm{O} 2<=>\mathrm{CO}+\mathrm{HO} 2$ ', [3.000000e+12, 0.0, 0.0])

falloff_reaction $(' \mathrm{CO}+\mathrm{O}(+\mathrm{M})<=>\mathrm{CO} 2(+\mathrm{M})$ ',

$\mathrm{kf}=[9.640000 \mathrm{e}+10,0.0,3800.0]$,

$\mathrm{kf} 0=[2.070000 \mathrm{e}+26,-3.34,7610.0]$,

efficiencies='AR:0.5 CO:1.5 CO2:2.0 H2:2.0 H2O:12.0 ')

reaction(' $\left.\mathrm{CO}+\mathrm{OH}<=>\mathrm{CO} 2+\mathrm{H}^{\prime},[9.600000 \mathrm{e}+11,0.14,7352.0]\right)$

reaction(' $\left.\mathrm{CO}+\mathrm{OH}<=>\mathrm{CO} 2+\mathrm{H}^{\prime},[7.320000 \mathrm{e}+10,0.03,-16.0]\right)$

reaction('CO + $\left.\mathrm{HO} 2<=>\mathrm{CO} 2+\mathrm{OH}^{\prime},[3.000000 \mathrm{e}+13,0.0,23000.0]\right)$

reaction('CO + H2O $<=>\mathrm{CO} 2+\mathrm{H} 2$ ', [2.000000e+11, 0.0, 38000.0])

falloff_reaction('CH3 + H $(+\mathrm{M})<=>\mathrm{CH} 4(+\mathrm{M})$ ',

$\mathrm{kf}=[1.200000 \mathrm{e}+15,-0.4,0.0]$,

$\mathrm{kf0}=[6.400000 \mathrm{e}+23,-1.8,0.0]$,

efficiencies='CO:2.0 CO2:3.0 H2:2.0 H2O:5.0 ',

falloff=SRI $(\mathrm{A}=0.45, \mathrm{~B}=797.0, \mathrm{C}=979.0, \mathrm{D}=1.0, \mathrm{E}=0.0))$

falloff_reaction('2 CH3 (+M) $<=>$ C2H6 (+M)',

$\mathrm{kf}=[2.500000 \mathrm{e}+13,0.0,0.0]$,

$\mathrm{kf0}=[2.330000 \mathrm{e}+34,-5.03,-1200.0]$,

efficiencies='CO:2.0 CO2:3.0 H2:2.0 H2O:5.0 ',

falloff $=\operatorname{Troe}(\mathrm{A}=0.38, \mathrm{~T} 3=73.0, \mathrm{~T} 1=1180.0, \mathrm{~T} 2=0.0))$

falloff_reaction('C2H5 + H $(+\mathrm{M})<=>$ C2H6 $(+\mathrm{M})$ ',

$\mathrm{kf}=[5.210000 \mathrm{e}+17,-0.99,1580.0]$,

$\mathrm{kf0}=[1.990000 \mathrm{e}+41,-7.08,6685.0]$,

efficiencies='AR:0.7 C2H6:3.0 CH4:2.0 CO:1.5 H2O:6.0 ',

falloff $=\operatorname{Troe}(\mathrm{A}=0.8422, \mathrm{~T} 3=125.0, \mathrm{~T} 1=2219.0, \mathrm{~T} 2=6882.0))$ 
reaction('CH2CHCH2 $+\mathrm{H}<=>\mathrm{AC} 3 \mathrm{H} 4+\mathrm{H} 2$ ', [1.810000e+13, 0.0, 0.0])

falloff_reaction('CH2CHCH2 $+\mathrm{H}(+\mathrm{M})<=>\mathrm{C} 3 \mathrm{H} 6(+\mathrm{M})$ ',

$\mathrm{kf}=[1.000000 \mathrm{e}+14,0.0,0.0]$,

$\mathrm{kf0}=[1.330000 \mathrm{e}+60,-12.0,5967.8]$,

efficiencies='AR:0.7 C2H6:3.0 CH4:2.0 CO:1.5 CO2:2.0 H2:2.0 H2O:6.0 ',

falloff $=\operatorname{Troe}(\mathrm{A}=0.02, \mathrm{~T} 3=1097.0, \mathrm{~T} 1=10970.0, \mathrm{~T} 2=6860.0))$

falloff_reaction $(' \mathrm{C} 2 \mathrm{H} 5+\mathrm{CH} 3(+\mathrm{M})<=>\mathrm{C} 3 \mathrm{H} 8(+\mathrm{M})$ ',

$\mathrm{kf}=[9.600000 \mathrm{e}+14,-0.5,0.0]$,

$\mathrm{kf0}=[6.800000 \mathrm{e}+61,-13.42,6000.0]$,

falloff $=\operatorname{Troe}(\mathrm{A}=1.0, \mathrm{~T} 3=1000.0, \mathrm{~T} 1=1434.0, \mathrm{~T} 2=5329.0))$

falloff_reaction('C3H3 $+\mathrm{H}(+\mathrm{M})<=>\mathrm{AC} 3 \mathrm{H} 4(+\mathrm{M})$ ',

$\mathrm{kf}=[1.000000 \mathrm{e}+17,-0.8,315.0]$,

$\mathrm{kf0}=[3.500000 \mathrm{e}+33,-4.9,2225.0]$,

efficiencies='C2H6:4.3 CH4:2.9 CO:2.1 CO2:2.9 H2:2.9 H2O:8.6 ',

falloff $=\operatorname{Troe}(\mathrm{A}=0.709, \mathrm{~T} 3=134.0, \mathrm{~T} 1=1784.0, \mathrm{~T} 2=5740.0))$

falloff_reaction('C3H3 $+\mathrm{H}(+\mathrm{M})<=>$ PC3H4 $(+\mathrm{M})$ ',

$\mathrm{kf}=[1.000000 \mathrm{e}+17,-0.8,315.0]$,

$\mathrm{kf} 0=[3.500000 \mathrm{e}+33,-4.9,2225.0]$,

efficiencies='C2H6:4.3 CH4:2.9 CO:2.1 CO2:2.9 H2:2.9 H2O:8.6 ',

falloff $=\operatorname{Troe}(\mathrm{A}=0.709, \mathrm{~T} 3=134.0, \mathrm{~T} 1=1784.0, \mathrm{~T} 2=5740.0))$

falloff_reaction $(' \mathrm{C} 2 \mathrm{H} 3+\mathrm{CH} 3(+\mathrm{M})<=>\mathrm{C} 3 \mathrm{H} 6(+\mathrm{M})$ ',

$\mathrm{kf}=[2.500000 \mathrm{e}+13,0.0,0.0]$,

$\mathrm{kf0}=[4.270000 \mathrm{e}+58,-11.94,9770.0]$,

efficiencies='AR:0.7 C2H6:3.0 CH4:2.0 CO:1.5 CO2:2.0 H2:2.0 H2O:6.0 ',

falloff $=$ Troe $(\mathrm{A}=0.175, \mathrm{~T} 3=1341.0, \mathrm{~T} 1=60000.0, \mathrm{~T} 2=10140.0))$

reaction('C4H4 $\Leftrightarrow>\mathrm{C} 2 \mathrm{H}+\mathrm{C} 2 \mathrm{H} 3$ ', [1.000000e+16, 0.0, 105000.0])

reaction('NC4H8 $<=>\mathrm{CH} 2 \mathrm{CHCH} 2+\mathrm{CH} 3$ ', [2.000000e+16, 0.0, 78000.0])

falloff_reaction('H + SC4H7 $(+\mathrm{M})<=>\mathrm{NC} 4 \mathrm{H} 8(+\mathrm{M})$ ',

$\mathrm{kf}=[5.000000 \mathrm{e}+13,0.0,0.0]$,

$\mathrm{kf} 0=[1.330000 \mathrm{e}+60,-12.0,5967.8]$,

efficiencies='AR:0.7 C2H6:3.0 CH4:2.0 CO:1.5 CO2:2.0 H2:2.0 H2O:6.0 ',

falloff $=\operatorname{Troe}(\mathrm{A}=0.02, \mathrm{~T} 3=1097.0, \mathrm{~T} 1=10970.0, \mathrm{~T} 2=6860.0))$

reaction(' $\left.\mathrm{C} 2 \mathrm{H} 2+\mathrm{C} 2 \mathrm{H} 3<=>\mathrm{C} 4 \mathrm{H} 4+\mathrm{H}^{\prime},[2.500000 \mathrm{e}+14,-0.71,6700.0]\right)$

reaction('C4H5 $\Leftrightarrow>\mathrm{C} 2 \mathrm{H} 2+\mathrm{C} 2 \mathrm{H} 3$ ', [7.500000e+12, 0.0, 40000.0])

reaction(' $\mathrm{C} 2 \mathrm{H} 3+\mathrm{H}<=>\mathrm{C} 2 \mathrm{H} 2+\mathrm{H} 2$ ', [3.000000e+13, 0.0, 0.0])

reaction('C2H4 + H2 $\Leftrightarrow=>\mathrm{C} 2 \mathrm{H} 5+\mathrm{H}$ ', [1.000000e+14, 0.0, 65000.0])

reaction(' $\mathrm{C} 2 \mathrm{H} 3+\mathrm{CH} 3<=>\mathrm{C} 2 \mathrm{H} 2+\mathrm{CH} 4$ ', [1.333000e+13, 0.0, 0.0])

reaction $(' \mathrm{C} 3 \mathrm{H} 3+\mathrm{CH} 2 \mathrm{CHCH} 2=>\mathrm{C} 2 \mathrm{H} 2+\mathrm{C} 4 \mathrm{H} 6$ ', $[5.000000 \mathrm{e}+11,0.0,0.0])$

falloff_reaction $(' \mathrm{C} 2 \mathrm{H} 2+\mathrm{H}(+\mathrm{M})<=>\mathrm{C} 2 \mathrm{H} 3(+\mathrm{M})$ ',

$\mathrm{kf}=[1.000000 \mathrm{e}+13,0.0,2770.0]$,

kf0 $=[3.900000 \mathrm{e}+16,0.0,-560.0]$,

efficiencies='CO:2.0 CO2:3.0 H2:2.0 H2O:5.0 ')

falloff_reaction $(' \mathrm{C} 2 \mathrm{H} 4+\mathrm{H}(+\mathrm{M})<=>\mathrm{C} 2 \mathrm{H} 5(+\mathrm{M})$ ', 
$\mathrm{kf}=[1.770000 \mathrm{e}+13,0.0,2110.0]$,

$\mathrm{kf0}=[4.600000 \mathrm{e}+18,0.0,1070.0]$,

efficiencies='CO:2.0 CO2:3.0 H2:2.0 H2O:5.0 ',

falloff $=\operatorname{Troe}(\mathrm{A}=1.0, \mathrm{~T} 3=1 \mathrm{e}-15, \mathrm{~T} 1=95.0, \mathrm{~T} 2=200.0))$

falloff_reaction('AC3H4 + H $(+\mathrm{M})<=>\mathrm{CH} 2 \mathrm{CHCH} 2(+\mathrm{M})$ ',

$\mathrm{kf}=[1.200000 \mathrm{e}+11,0.69,3007.0]$,

$\mathrm{kf0}=[5.660000 \mathrm{e}+33,-5.0,4448.0])$

falloff_reaction('AC3H4 + H $(+\mathrm{M})<=>\mathrm{CH} 2 \mathrm{CCH} 3(+\mathrm{M})$ ',

$\mathrm{kf}=[8.490000 \mathrm{e}+12,0.0,2000.0]$,

$\mathrm{kf0}=[1.110000 \mathrm{e}+34,-5.0,4448.0])$

reaction('H + PC3H4 $\Leftrightarrow=>\mathrm{CH} 2 \mathrm{CCH} 3$ ', [5.000000e+13, 0.0, 4000.0])

reaction('CH2CCH3 $\Leftrightarrow=>\mathrm{C} 2 \mathrm{H} 2+\mathrm{CH} 3$ ', [2.000000e+13, 0.0, 38000.0])

reaction('IC3H7 <=> NC3H7', [2.000000e+12, 0.0, 42000.0])

reaction('NC3H7 $\Leftrightarrow=>\mathrm{C} 2 \mathrm{H} 4+\mathrm{CH} 3$ ', [1.000000e+13, 0.0, 32000.0])

reaction('NC3H7 $\Leftrightarrow>\mathrm{C} 3 \mathrm{H} 6+\mathrm{H}$ ', [1.000000e+13, 0.0, 39500.0])

reaction('C4H2 + H $<=>$ C4H3', [2.500000e+14, 0.0, 3016.0])

reaction('C4H5 $\Leftrightarrow=>\mathrm{C} 4 \mathrm{H} 4+\mathrm{H}$ ', [5.000000e+12, 0.0, 44000.0])

reaction('C4H4 + H $\Leftrightarrow=>\mathrm{C} 2 \mathrm{H}+\mathrm{C} 2 \mathrm{H} 4$ ', [2.000000e+13, 0.0, 2000.0])

reaction('CH2C3H5 $\Leftrightarrow=\mathrm{C} 2 \mathrm{H} 3+\mathrm{C} 2 \mathrm{H} 4$ ', [2.500000e+13, 0.0, 38000.0])

reaction('2 CH3 $\Leftrightarrow=>\mathrm{C} 2 \mathrm{H} 5+\mathrm{H}$ ', [1.400000e+14, 0.0, 14000.0])

reaction('H + PC3H4 $<=>\mathrm{C} 2 \mathrm{H} 2+\mathrm{CH} 3$ ', [2.000000e+05, 2.5, 1000.0])

reaction('C2 $\mathrm{H} 6<=>\mathrm{C} 2 \mathrm{H} 4+\mathrm{H} 2$ ', [3.000000e+13, 0.0, 71000.0])

falloff_reaction $(\mathrm{CH} 3 \mathrm{OH}(+\mathrm{M})<=>\mathrm{CH} 3+\mathrm{OH}(+\mathrm{M})$ ',

$\mathrm{kf}=[7.000000 \mathrm{e}+20,-1.3,92000.0]$,

$\mathrm{kf0}=[1.250000 \mathrm{e}+14,0.85,67000.0])$

reaction('CH3CHO $\left.\Leftrightarrow>\mathrm{CH} 3+\mathrm{HCO}^{\prime},[1.500000 \mathrm{e}+16,0.0,85000.0]\right)$

reaction $(' \mathrm{C} 3 \mathrm{H} 6+\mathrm{OH}=>\mathrm{CH} 3+\mathrm{CH} 3 \mathrm{CHO}$ ', [2.000000e+12, 0.0, 0.0])

reaction ('C2 $\left.2 \mathrm{H} 2+\mathrm{O} 2 \Leftrightarrow=>\mathrm{HCCO}+\mathrm{OH}^{\prime},[2.000000 \mathrm{e}+07,1.5,30000.0]\right)$

reaction('CH4 + O2 $\Leftrightarrow=>\mathrm{CH} 3+\mathrm{HO} 2$ ', [9.000000e+13, 0.0, 56000.0])

reaction('CH2O + $\mathrm{O} 2<=>\mathrm{HCO}+\mathrm{HO} 2$ ', [1.300000e+14, 0.0, 41000.0])

three_body_reaction('HCO $+\mathrm{M}<=>\mathrm{CO}+\mathrm{H}+\mathrm{M}^{\prime}$, [1.200000e+17, $\left.-1.0,17000.0\right]$,

efficiencies='CH4:2.8 CO:1.9 CO2:3.0 H2:1.9 H2O:5.0 ')

falloff_reaction('CH3O $(+\mathrm{M})<=>\mathrm{CH} 2 \mathrm{O}+\mathrm{H}(+\mathrm{M})$ ',

$\mathrm{kf}=[6.000000 \mathrm{e}+11,0.0,18000.0]$,

$\mathrm{kf0}=[1.200000 \mathrm{e}+25,-2.7,30600.0])$

three_body_reaction('CH2OH $+\mathrm{M} \Leftrightarrow=>\mathrm{CH} 2 \mathrm{O}+\mathrm{H}+\mathrm{M}^{\prime}$, [3.750000e+14, 0.0, 25000.0])

reaction('CH3CO $\left.\Leftrightarrow>\mathrm{CH} 2 \mathrm{CO}+\mathrm{H}^{\prime},[1.000000 \mathrm{e}+14,0.0,49000.0]\right)$

three_body_reaction('CH3CO $+\mathrm{M}<=>\mathrm{CH} 3+\mathrm{CO}+\mathrm{M}$ ', [2.500000e+15, 0.0, 14400.0])

reaction('CH2CO + H => CH3 + CO', [1.000000e+06, 2.0, 2000.0])

reaction('CH2CO + $\left.\mathrm{H} \Leftrightarrow=>\mathrm{H} 2+\mathrm{HCCO}^{\prime},[3.600000 \mathrm{e}+14,0.0,8600.0]\right)$

reaction $(' \mathrm{CH} 3 \mathrm{OH}+\mathrm{H}=>\mathrm{CH} 3+\mathrm{H} 2 \mathrm{O}$ ', [6.500000e+11, 0.0, 5300.0])

reaction(' $\left.\mathrm{C} 2 \mathrm{H} 4+\mathrm{O}<=>\mathrm{CH} 3+\mathrm{HCO}^{\prime},[5.000000 \mathrm{e}+06,1.88,200.0]\right)$

reaction('AC3H4 $\left.+\mathrm{O}=>\mathrm{C} 2 \mathrm{H} 4+\mathrm{CO}^{\prime},[2.000000 \mathrm{e}+13,0.0,0.0]\right)$ 
reaction('C3H6 + O => C2H5 + HCO', [1.000000e+13, 0.0, 3000.0])

reaction $(' \mathrm{CH} 2 \mathrm{O}+\mathrm{O}=>\mathrm{CO} 2+2 \mathrm{H}$ ', [2.000000e+13, 0.0, 5000.0])

reaction $\left(' \mathrm{CH} 3 \mathrm{CHO}+\mathrm{O}=>\mathrm{CH} 3+\mathrm{CO} 2+\mathrm{H}^{\prime},[2.000000 \mathrm{e}+13,0.0,3000.0]\right)$

reaction(' $\mathrm{CH} 2 \mathrm{CO}+\mathrm{O}=>2 \mathrm{HCO}$ ', [2.000000e+13, 0.0, 2300.0])

reaction $\left(' \mathrm{C} 2 \mathrm{H} 2+\mathrm{OH}=>\mathrm{CH} 3+\mathrm{CO}^{\prime},[1.500000 \mathrm{e}+11,0.0,0.0]\right)$

reaction $(' \mathrm{CH} 2 \mathrm{CO}+\mathrm{OH}=>\mathrm{CH} 3+\mathrm{CO} 2$ ', [1.000000e+13, 0.0, 0.0])

reaction $\left(' \mathrm{CH} 2 \mathrm{CO}+\mathrm{OH}=>\mathrm{CH} 2 \mathrm{O}+\mathrm{HCO}^{\prime},[1.000000 \mathrm{e}+13,0.0,0.0]\right)$

reaction $\left(' \mathrm{C} 4 \mathrm{H} 4+\mathrm{OH}=>\mathrm{CH} 2 \mathrm{CHCH} 2+\mathrm{CO}^{\prime},[3.000000 \mathrm{e}+13,0.0,0.0]\right)$

reaction $\left(' \mathrm{C} 2 \mathrm{H} 2+\mathrm{HO} 2=>\mathrm{CH} 2 \mathrm{O}+\mathrm{HCO}^{\prime},[5.000000 \mathrm{e}+12,0.0,15000.0]\right)$

reaction $\left(' \mathrm{C} 2 \mathrm{H} 2+\mathrm{O} 2=>\mathrm{CH} 2 \mathrm{O}+\mathrm{CO}^{\prime}\right.$, [3.000000e+11, 0.0, 26000.0])

reaction $(' \mathrm{CH} 2 \mathrm{CO}+\mathrm{O} 2=>\mathrm{CH} 2 \mathrm{O}+\mathrm{CO} 2$ ', [1.000000e+14, 0.0, 37000.0])

reaction(' $\left.\mathrm{C} 2 \mathrm{H} 2+\mathrm{O} 2=>2 \mathrm{HCO}^{\prime},[3.000000 \mathrm{e}+11,0.0,27000.0]\right)$

reaction ('C2 $\left.\mathrm{H} 4+\mathrm{O} 2=>\mathrm{CH} 3 \mathrm{O}+\mathrm{HCO}^{\prime},[1.000000 \mathrm{e}+14,0.0,43000.0]\right)$

reaction(' $\mathrm{CH} 2 \mathrm{OH}+\mathrm{O} 2 \Leftrightarrow=>\mathrm{CH} 2 \mathrm{O}+\mathrm{HO}_{2}$, [6.000000e+12, 0.0, 0.0])

reaction $(' \mathrm{C} 2 \mathrm{H} 5+\mathrm{O} 2=>\mathrm{C} 2 \mathrm{H} 4+\mathrm{HO} 2$ ', [1.000000e+12, 0.0, 3000.0])

reaction('C2H3 + $\left.\mathrm{O} 2 \Leftrightarrow=>\mathrm{CH} 2 \mathrm{CHO}+\mathrm{O}^{\prime},[7.500000 \mathrm{e}+14,-0.61,5260.0]\right)$

reaction $\left(' \mathrm{C} 2 \mathrm{H} 3+\mathrm{O} 2=>\mathrm{CH} 2 \mathrm{O}+\mathrm{HCO}^{\prime},[1.000000 \mathrm{e}+12,0.0,4000.0]\right)$

reaction $(' \mathrm{C} 2 \mathrm{H} 3+\mathrm{O} 2=>\mathrm{CH} 2 \mathrm{CO}+\mathrm{OH}$ ', [6.000000e+11, 0.0, 1000.0])

three_body_reaction(' $\left.\mathrm{CH} 3+\mathrm{O}+\mathrm{M}=>\mathrm{CH} 3 \mathrm{O}+\mathrm{M}^{\prime},[5.000000 \mathrm{e}+16,0.0,0.0]\right)$

reaction ('C2 $\left.\mathrm{H} 5+\mathrm{O}=>0.3 \mathrm{C} 2 \mathrm{H} 4+0.35 \mathrm{CH} 2 \mathrm{O}+0.35 \mathrm{CH} 3+0.35 \mathrm{CH} 3 \mathrm{CHO}+0.35 \mathrm{H}+0.3 \mathrm{OH}^{\prime},[5.000000 \mathrm{e}+13,0.0,0.0]\right)$

reaction $(' \mathrm{CH} 2 \mathrm{CHCH} 2+\mathrm{O}=>\mathrm{C} 2 \mathrm{H} 3+\mathrm{CH} 2 \mathrm{O}$ ', [3.250000e+13, 0.0, 0.0])

reaction $\left(' \mathrm{CH} 3+\mathrm{OH}<=>\mathrm{CH} 2 \mathrm{OH}+\mathrm{H}^{\prime},[1.100000 \mathrm{e}+13,0.0,6300.0]\right)$

reaction $(' \mathrm{CH} 3+\mathrm{OH} \Leftrightarrow=>\mathrm{CH} 2 \mathrm{O}+\mathrm{H} 2$ ', $[6.000000 \mathrm{e}+12,0.0,0.0])$

reaction(' $\left.\mathrm{CH} 3+\mathrm{OH}<=>\mathrm{CH} 4+\mathrm{O}^{\prime},[2.000000 \mathrm{e}+12,0.0,8000.0]\right)$

reaction('CH3 + $\left.\mathrm{HO} 2 \Leftrightarrow=>\mathrm{CH} 3 \mathrm{O}+\mathrm{OH}^{\prime},[6.000000 \mathrm{e}+12,0.0,0.0]\right)$

reaction $(' \mathrm{C} 2 \mathrm{H} 5+\mathrm{HO} 2=>\mathrm{CH} 2 \mathrm{O}+\mathrm{CH} 3+\mathrm{OH}$, [5.000000e+12, 0.0, 0.0])

reaction $\left(' \mathrm{CH} 2 \mathrm{CHCH} 2+\mathrm{HO} 2=>\mathrm{C} 2 \mathrm{H} 3+\mathrm{CH} 2 \mathrm{O}+\mathrm{OH}^{\prime},[3.500000 \mathrm{e}+11,0.0,0.0]\right)$

reaction $(' \mathrm{H}+\mathrm{HCO} \Leftrightarrow=>\mathrm{CO}+\mathrm{H} 2$ ', [5.000000e+13, 0.0, 0.0])

reaction('HCO $+\mathrm{OH}<=>\mathrm{CO}+\mathrm{H}_{2} \mathrm{O}$ ', [5.000000e+13, 0.0, 0.0])

reaction $\left(' \mathrm{HCO}+\mathrm{HO} 2=>\mathrm{CO} 2+\mathrm{H}+\mathrm{OH}^{\prime},[3.000000 \mathrm{e}+13,0.0,0.0]\right)$

reaction $\left(' \mathrm{CH} 3+\mathrm{HCO} \Leftrightarrow=>\mathrm{CH} 4+\mathrm{CO}^{\prime},[1.000000 \mathrm{e}+13,0.0,0.0]\right)$

reaction $\left(' \mathrm{CH} 2 \mathrm{OH}+\mathrm{CH} 3=>\mathrm{CH} 2 \mathrm{O}+\mathrm{CH}_{4}\right.$ ', [1.500000e+13, 0.0, 0.0])

reaction('CH2CHO $<=>$ CH3CO', [2.000000e+11, 0.0, 32000.0])

reaction('CH2CHO $\left.\Leftrightarrow=\mathrm{CH} 2 \mathrm{CO}+\mathrm{H}^{\prime},[6.000000 \mathrm{e}+13,0.0,41000.0]\right)$

reaction (' $\left.\mathrm{C} 2 \mathrm{H} 4+\mathrm{O} \Rightarrow \mathrm{CH} 2 \mathrm{CHO}+\mathrm{H}^{\prime},[1.000000 \mathrm{e}+13,0.0,3000.0]\right)$

reaction $\left(' \mathrm{C} 2 \mathrm{H} 2+\mathrm{OH}=>\mathrm{CH} 2 \mathrm{CHO}^{\prime},[5.000000 \mathrm{e}+11,0.0,0.0]\right)$

reaction(' $\mathrm{C} 4 \mathrm{H} 6+\mathrm{OH}=>0.2 \mathrm{C} 2 \mathrm{H} 3+0.25 \mathrm{C} 2 \mathrm{H} 4+0.25 \mathrm{CH} 2 \mathrm{CHO}+0.55 \mathrm{CH} 2 \mathrm{O}+0.2 \mathrm{CH} 3 \mathrm{CHO}+0.55 \mathrm{CHCHCH} 3$,

$[5.000000 \mathrm{e}+12,0.0,0.0])$

reaction('CH2S + H2 $\left.<=>\mathrm{CH} 3+\mathrm{H}^{\prime},[7.200000 \mathrm{e}+13,0.0,0.0]\right)$

reaction $(' \mathrm{CH} 3+\mathrm{OH}<=>\mathrm{CH} 2 \mathrm{~S}+\mathrm{H} 2 \mathrm{O}$ ', [2.000000e+13, 0.0, 0.0])

reaction $\left(' \mathrm{CH} 2 \mathrm{O}+\mathrm{CH} 3<=>\mathrm{CH} 3 \mathrm{CHO}+\mathrm{H}^{\prime},[2.000000 \mathrm{e}+11,0.0,7600.0]\right)$

reaction('CH2 + $\left.\mathrm{O} 2<=>\mathrm{CH} 2 \mathrm{O}+\mathrm{O}^{\prime},[5.000000 \mathrm{e}+13,0.0,9000.0]\right)$

reaction('CH2 + O2 $<=>\mathrm{CO}+\mathrm{H}_{2} \mathrm{O}$ ', [1.600000e+13, 0.0, 1500.0]) 
reaction('CH2 + O2 $\left.\Leftrightarrow=\mathrm{CO}+\mathrm{H}+\mathrm{OH}^{\prime},[1.700000 \mathrm{e}+13,0.0,1500.0]\right)$

reaction('CH2 $+\mathrm{O} 2 \Leftrightarrow=>\mathrm{CO} 2+2 \mathrm{H}$ ', [1.320000e+13, 0.0, 1500.0])

three_body_reaction('CH2S $+\mathrm{M} \Leftrightarrow=>\mathrm{CH} 2+\mathrm{M}^{\prime},[1.000000 \mathrm{e}+13,0.0,0.0]$,

efficiencies='C2H2:4.0 H:20.0 H2O:3.0 ')

reaction(' $\left.\mathrm{C} 2 \mathrm{H} 2+\mathrm{O}<=>\mathrm{CH} 2+\mathrm{CO}^{\prime},[3.500000 \mathrm{e}+03,2.8,500.0]\right)$

reaction(' $\left.\mathrm{C} 2 \mathrm{H} 2+\mathrm{O}<=>\mathrm{H}+\mathrm{HCCO}^{\prime},[5.000000 \mathrm{e}+06,2.0,1900.0]\right)$

falloff_reaction $(' \mathrm{CH} 2 \mathrm{CO}(+\mathrm{M})<=>\mathrm{CH} 2+\mathrm{CO}(+\mathrm{M})$ ',

$\mathrm{kf}=[1.500000 \mathrm{e}+14,0.0,76000.0]$,

$\mathrm{kf0}=[5.500000 \mathrm{e}+15,0.0,59270.0])$

reaction(' $\mathrm{CH} 2 \mathrm{CO}+\mathrm{O}<=>\mathrm{CH} 2+\mathrm{CO} 2$ ', [1.500000e+12, 0.0, 1350.0])

reaction(' $\mathrm{HCCO}<=>\mathrm{CH}+\mathrm{CO}^{\prime},[6.500000 \mathrm{e}+12,0.0,59000.0]$ )

reaction $\left(' \mathrm{H}+\mathrm{HCCO} \Leftrightarrow=>\mathrm{CH} 2 \mathrm{~S}+\mathrm{CO}^{\prime},[1.500000 \mathrm{e}+14,0.0,0.0]\right)$

reaction('HCCO $+\mathrm{O} \Leftrightarrow=>2 \mathrm{CO}+\mathrm{H}$ ', [9.600000e+13, 0.0,600.0])

reaction('HCCO $\left.+\mathrm{O} 2 \Leftrightarrow=>2 \mathrm{CO}+\mathrm{OH}^{\prime},[1.600000 \mathrm{e}+12,0.0,830.0]\right)$

reaction('HCCO + O2 $\left.\Leftrightarrow=>\mathrm{CO} 2+\mathrm{HCO}^{\prime},[2.400000 \mathrm{e}+11,0.0,-854.0]\right)$

reaction(' $\left.\mathrm{C} 2 \mathrm{H}+\mathrm{O} 2<=>\mathrm{HCCO}+\mathrm{O}^{\prime},[2.300000 \mathrm{e}+13,0.0,0.0]\right)$

reaction('C4H2 + O $\left.=>\mathrm{C} 3 \mathrm{H} 2+\mathrm{CO}^{\prime},[2.700000 \mathrm{e}+13,0.0,1660.0]\right)$

reaction('C4H2 + $\left.\mathrm{OH} \Leftrightarrow=>\mathrm{C} 3 \mathrm{H} 2+\mathrm{HCO}^{\prime},[3.000000 \mathrm{e}+12,0.0,0.0]\right)$

reaction(' $\mathrm{C} 3 \mathrm{H} 3+\mathrm{H}<=>\mathrm{C} 3 \mathrm{H} 2+\mathrm{H} 2$ ', [1.500000e+13, 0.0, 3000.0])

reaction(' $\mathrm{C} 3 \mathrm{H} 3+\mathrm{O}<=>\mathrm{C} 2 \mathrm{H}+\mathrm{CH} 2 \mathrm{O}$ ', [3.000000e+13, 0.0, 0.0])

reaction(' $\mathrm{C} 3 \mathrm{H} 3+\mathrm{OH}<=>\mathrm{C} 3 \mathrm{H} 2+\mathrm{H} 2 \mathrm{O}$ ', [1.500000e+13, 0.0, 3000.0])

reaction(' $\left.\mathrm{C} 3 \mathrm{H} 3+\mathrm{O} 2 \Leftrightarrow=>\mathrm{CH} 2 \mathrm{CO}+\mathrm{HCO}^{\prime},[2.000000 \mathrm{e}+10,0.0,2840.0]\right)$

reaction('C3H3 + $\mathrm{HCO}<=>\mathrm{CO}+\mathrm{PC} 3 \mathrm{H} 4$ ', [2.500000e+13, 0.0, 0.0])

reaction $\left(' \mathrm{C} 3 \mathrm{H} 2+\mathrm{O} 2<=>\mathrm{HCCO}+\mathrm{HCO}^{\prime},[4.000000 \mathrm{e}+13,0.0,0.0]\right)$

reaction('C3H6 + $\mathrm{H} \Leftrightarrow=>\mathrm{CH} 2 \mathrm{CHCH} 2+\mathrm{H} 2$ ', [1.000000e+07, 2.0, 4200.0])

reaction('C2H4 $+\mathrm{OH}<=>\mathrm{C} 2 \mathrm{H} 3+\mathrm{H} 2 \mathrm{O}$ ', [2.000000e+13, 0.0, 6000.0])

reaction(' $\mathrm{CH} 3 \mathrm{OH}+\mathrm{H}<=>\mathrm{CH} 3 \mathrm{O}+\mathrm{H} 2$ ', [9.000000e+12, 0.0, 6100.0])

reaction ('CH3OH $+\mathrm{H}<=>\mathrm{CH} 2 \mathrm{OH}+\mathrm{H} 2$ ', [3.200000e+13, 0.0, 6100.0])

reaction('CH2O + H $\Leftrightarrow=\mathrm{H} 2+\mathrm{HCO}^{\prime}$, [4.500000e+14, 0.0, 7500.0])

reaction(' $\mathrm{CH} 3 \mathrm{CHO}+\mathrm{H} \Leftrightarrow=>\mathrm{CH} 3 \mathrm{CO}+\mathrm{H} 2$ ', [4.500000e+14, 0.0, 7500.0])

reaction('AC3H4 + $\mathrm{H}<=>\mathrm{C} 3 \mathrm{H} 3+\mathrm{H} 2$ ', [5.000000e+07, 2.0, 5000.0])

reaction(' $\mathrm{H}+\mathrm{PC} 3 \mathrm{H} 4<=>\mathrm{C} 3 \mathrm{H} 3+\mathrm{H} 2$ ', [1.000000e+07, 2.0, 5000.0])

reaction('OH + PC3H4 $\left.<=>\mathrm{C} 3 \mathrm{H} 3+\mathrm{H}_{2} \mathrm{O}^{\prime},[8.000000 \mathrm{e}+06,2.0,1000.0]\right)$

reaction $\left(' \mathrm{H}+\mathrm{H} 2 \mathrm{O}<=>\mathrm{H} 2+\mathrm{OH}^{\prime},[4.800000 \mathrm{e}+10,1.0,19000.0]\right)$

reaction('H + H2O2 $<=>\mathrm{H} 2 \mathrm{O}+\mathrm{OH}^{\prime}$, [2.410000e+13, 0.0, 3970.0])

reaction('H + H2O2 $<=>\mathrm{H} 2+\mathrm{HO} 2$ ', [6.025000e+13, 0.0, 7950.0])

reaction('CH4 + $\mathrm{H}<=>\mathrm{CH} 3+\mathrm{H} 2$ ', [3.000000e+07, 2.0, 10000.0])

reaction('C2H6 + H $\Leftrightarrow=>\mathrm{C} 2 \mathrm{H} 5+\mathrm{H} 2$ ', [1.430000e+14, 0.0, 10500.0])

reaction('C3H8 + H $<=>\mathrm{H} 2+\mathrm{NC} 3 \mathrm{H} 7$ ', [1.270000e+14, 0.0, 10500.0])

reaction('C3H8 + H $<=>\mathrm{H} 2+\mathrm{IC} 3 \mathrm{H} 7$ ', [3.200000e+13, 0.0, 7900.0])

reaction('CH2O + $\left.\mathrm{HO} 2=>\mathrm{H} 2 \mathrm{O} 2+\mathrm{HCO}^{\prime},[5.200000 \mathrm{e}+12,0.0,13000.0]\right)$

reaction $\left(' \mathrm{CH} 3 \mathrm{OH}+\mathrm{OH}=>\mathrm{CH} 2 \mathrm{OH}+\mathrm{H}_{2} \mathrm{O}\right.$ ', [9.200000e+04, 2.53, -1000.0])

reaction $(' \mathrm{CH} 3 \mathrm{OH}+\mathrm{HO} 2=>\mathrm{CH} 2 \mathrm{OH}+\mathrm{H} 2 \mathrm{O} 2$ ', [8.000000e+13, 0.0, 19400.0]) 
reaction(' $\mathrm{C} 3 \mathrm{H} 6+\mathrm{OH}=>\mathrm{CH} 2 \mathrm{CHCH} 2+\mathrm{H} 2 \mathrm{O}$ ', [1.680000e+06, 2.0, -432.1])

reaction(' $\mathrm{C} 2 \mathrm{H} 2+\mathrm{OH}<=>\mathrm{C} 2 \mathrm{H}+\mathrm{H} 2 \mathrm{O}$ ', [3.370000e+07, 2.0, 14000.0])

reaction('CYC5H6 $\Leftrightarrow=>$ CYC5H5 + H', [1.500000e+15, 0.0, 81500.0])

reaction('CYC5H6 + OH $\left.=>\mathrm{C} 4 \mathrm{H} 6+\mathrm{HCO}^{\prime},[8.000000 \mathrm{e}+12,0.0,0.0]\right)$

reaction $\left(' \mathrm{CYC} 5 \mathrm{H} 6+\mathrm{OH}=>\mathrm{C} 2 \mathrm{H} 2+\mathrm{C} 2 \mathrm{H} 4+\mathrm{HCO}^{\prime},[2.000000 \mathrm{e}+12,0.0,0.0]\right)$

reaction ('CYC5H6 $+\mathrm{OH}=>\mathrm{C} 2 \mathrm{H} 2+\mathrm{CH} 2 \mathrm{CO}+\mathrm{CH} 3$ ', [2.000000e+13, 0.0, 0.0])

reaction('CYC5H6 + O $\left.=>\mathrm{C} 4 \mathrm{H} 6+\mathrm{CO}^{\prime},[1.000000 \mathrm{e}+13,0.0,0.0]\right)$

reaction('CYC5H6 + O => C4H5 + HCO', [1.500000e+13, 0.0, 0.0])

reaction('CYC5H6 + O $\Rightarrow$ AC3H4 + CH2CO', [3.000000e+12, 0.0, 0.0])

reaction('CYC5H6 + O $=>\mathrm{CH} 2 \mathrm{CO}+\mathrm{PC} 3 \mathrm{H} 4$ ', [3.000000e+12, 0.0, 0.0])

reaction $\left(' \mathrm{CYC} 5 \mathrm{H} 5+\mathrm{HO} 2=>\mathrm{C} 4 \mathrm{H} 5+\mathrm{CO}+\mathrm{OH}^{\prime},[3.300000 \mathrm{e}+13,0.0,0.0]\right)$

reaction('CYC5H5 + $\left.\mathrm{HO} 2=>\mathrm{C} 2 \mathrm{H}+\mathrm{C} 2 \mathrm{H} 4+\mathrm{CO}+\mathrm{OH}^{\prime},[1.500000 \mathrm{e}+13,0.0,0.0]\right)$

reaction('CYC5H5 $\left.+\mathrm{HO} 2=>\mathrm{C} 4 \mathrm{H} 4+\mathrm{HCO}+\mathrm{OH}^{\prime},[3.000000 \mathrm{e}+12,0.0,0.0]\right)$

reaction $\left(' \mathrm{CYC} 5 \mathrm{H} 5+\mathrm{OH}=>\mathrm{C} 2 \mathrm{H} 2+\mathrm{C} 2 \mathrm{H} 4+\mathrm{CO}^{\prime},[2.000000 \mathrm{e}+14,0.25,4350.0]\right)$

reaction('CYC5H5 + OH $\left.=>\mathrm{C} 3 \mathrm{H} 3+\mathrm{CH} 2 \mathrm{CO}+\mathrm{H}^{\prime},[3.500000 \mathrm{e}+13,0.25,4350.0]\right)$

reaction('CYC5H5 + OH => C4H6 + CO', [1.250000e+13, 0.25, 4350.0])

reaction ('CYC5H5 + O $\left.=>\mathrm{C} 4 \mathrm{H} 5+\mathrm{CO}^{\prime},[1.000000 \mathrm{e}+14,0.0,0.0]\right)$

reaction $\left(' \mathrm{CYC} 5 \mathrm{H} 5+\mathrm{O} 2=>\mathrm{C} 5 \mathrm{H} 5 \mathrm{O}+\mathrm{O}^{\prime},[1.500000 \mathrm{e}+11,0.0,16000.0]\right)$

reaction('C5H5O $=>$ CYC5H4O + H', [1.000000e+13, 0.0, 52000.0])

reaction('CYC5H6 + H => CYC5H5 + H2', [1.600000e+15, 0.0, 7925.0])

reaction('CYC5H5 + H2 => CYC5H6 + H', [1.800000e+14, 0.0, 30000.0])

reaction('CYC5H6 + OH => CYC5H5 + H2O', [1.000000e+14, 0.0, 1714.0])

reaction('CYC5H5 $=>\mathrm{C} 2 \mathrm{H} 2+\mathrm{C} 3 \mathrm{H} 3$ ', [2.000000e+12, 0.0, 68000.0])

reaction('CH3 + CYC5H5 => C2H3 + C4H5', [1.000000e+12, 0.0, 3000.0])

reaction $(' \mathrm{CH} 3+\mathrm{CYC} 5 \mathrm{H} 5=>\mathrm{C} 2 \mathrm{H} 2+\mathrm{C} 4 \mathrm{H} 6$ ', [1.500000e+12, 0.0, 3000.0])

reaction('2 CYC5H5 => C10H8 + 2 H', [1.000000e+12, 0.0, 6000.0])

reaction $(' \mathrm{CH} 3+\mathrm{CYC} 5 \mathrm{H} 5=>$ MCPTD', [1.000000e+13, 0.0, 3000.0])

reaction ('H + MCPTD => CH3 + CYC5H6', [1.500000e+13, 0.0, 2000.0])

reaction('C6H6 $<=>$ C6H5 + H', [1.500000e+17, 0.0, 114000.0])

reaction('C6H6 + OH $\left.=>\mathrm{CO}+\mathrm{CYC} 5 \mathrm{H} 6+\mathrm{H}^{\prime},[1.000000 \mathrm{e}+13,0.0,7000.0]\right)$

falloff_reaction('2 C3H3 $(+\mathrm{M})<=>\mathrm{C} 6 \mathrm{H} 6(+\mathrm{M})$ ',

$\mathrm{kf}=[3.000000 \mathrm{e}+12,0.0,0.0]$,

$\mathrm{kf0}=[5.000000 \mathrm{e}+17,0.0,0.0])$

reaction('2 $\mathrm{C} 3 \mathrm{H} 3 \Leftrightarrow=>\mathrm{C} 6 \mathrm{H} 5+\mathrm{H}$ ', [3.000000e+12, 0.0, 0.0])

reaction('C6H5 + O $\Rightarrow$ CO + CYC5H5', [1.000000e+14, 0.0, 0.0])

reaction('C6H6 + H $\Leftrightarrow=>\mathrm{C} 6 \mathrm{H} 5+\mathrm{H} 2$ ', [1.500000e+14, 0.0, 10000.0])

reaction('C10H8 + H => C4H4 + C6H5', [5.000000e+12, 0.0, 2500.0])

reaction('CH3 + O2 $<=>\mathrm{CH} 3 \mathrm{OO}$ ', [2.000000e+12, 0.0, 0.0])

reaction('CH3OO $\left.\Leftrightarrow=\mathrm{CH} 2 \mathrm{O}+\mathrm{OH}^{\prime},[1.500000 \mathrm{e}+13,0.0,47000.0]\right)$

reaction $(' \mathrm{CH} 3+\mathrm{CH} 3 \mathrm{OO}=>2 \mathrm{CH} 3 \mathrm{O}$ ', [3.000000e+13, 0.0, -1200.0])

three_body_reaction('CH3CHOH $\left.+\mathrm{M} \Leftrightarrow=>\mathrm{CH} 3 \mathrm{CHO}+\mathrm{H}+\mathrm{M}^{\prime},[1.800000 \mathrm{e}+24,-2.5,34200.0]\right)$

falloff_reaction('C2 $44+\mathrm{OH}(+\mathrm{M})<=>\mathrm{C} 2 \mathrm{H} 4 \mathrm{OH}(+\mathrm{M})$ ',

$\mathrm{kf}=[2.000000 \mathrm{e}+12,0.0,0.0]$, 
$\mathrm{kf} 0=[2.000000 \mathrm{e}+15,0.0,-10000.0]$,

falloff $=\operatorname{Troe}(\mathrm{A}=0.25, \mathrm{~T} 3=1 \mathrm{e}-30, \mathrm{~T} 1=1.0000000000000002 \mathrm{e}+30, \mathrm{~T} 2=0.0))$

reaction(' $\mathrm{CH} 3 \mathrm{CHOH}+\mathrm{O} 2 \Leftrightarrow=>\mathrm{CH} 3 \mathrm{CHO}+\mathrm{HO}_{2}$, [2.000000e+12, 0.0, 3000.0])

reaction('C3H6O $\Rightarrow>\mathrm{CH} 2 \mathrm{CHO}+\mathrm{CH} 3$ ', [5.000000e+16, 0.0, 73000.0])

reaction(' $\mathrm{C} 3 \mathrm{H} 6 \mathrm{O}=>\mathrm{C} 2 \mathrm{H} 4+\mathrm{CH} 2 \mathrm{O}$ ', [1.000000e+16, 0.0, 64000.0])

reaction('C3H6 + O => C3H6O', [7.000000e+12, 0.0, 5000.0])

reaction('C2H4O2 $\left.\Leftrightarrow=>\mathrm{CH} 2 \mathrm{OH}+\mathrm{HCO}^{\prime},[1.000000 \mathrm{e}+16,0.0,82000.0]\right)$

reaction(' $\mathrm{C} 2 \mathrm{H} 4 \mathrm{O} 2 \Leftrightarrow=>2 \mathrm{CH} 2 \mathrm{O}$ ', [5.000000e+12, 0.0, 60000.0])

reaction('C2H4O2 $<=>\mathrm{CH} 2 \mathrm{CO}+\mathrm{H} 2 \mathrm{O}$ ', [1.000000e+14, 0.0, 70000.0])

reaction('C2H2O2 $\Leftrightarrow=>2$ HCO', [3.000000e+16, 0.0, 71000.0])

reaction('C4H6O2 $\Leftrightarrow=>2 \mathrm{CH} 3 \mathrm{CO}$ ', [1.000000e+16, 0.0, 72000.0])

reaction('C6H6O3 $\left.=>\mathrm{C} 5 \mathrm{H} 4 \mathrm{O} 2+\mathrm{H}+\mathrm{HCO}^{\prime},[2.000000 \mathrm{e}+16,0.0,79000.0]\right)$

reaction('C6H6O3 $\Leftrightarrow=>\mathrm{C} 5 \mathrm{H} 4 \mathrm{O} 2+\mathrm{CH} 2 \mathrm{O}$ ', [5.000000e+12, 0.0, 59000.0])

reaction $\left(' \mathrm{C} 4 \mathrm{H} 3 \mathrm{O}=>\mathrm{C} 3 \mathrm{H} 3+\mathrm{CO}^{\prime},[1.500000 \mathrm{e}+12,0.0,38000.0]\right)$

reaction $(' \mathrm{C} 4 \mathrm{H} 3 \mathrm{O}=>\mathrm{C} 2 \mathrm{H} 2+\mathrm{HCCO}$ ', [1.500000e+12, 0.0, 38000.0])

reaction $(' \mathrm{C} 4 \mathrm{H} 3 \mathrm{O}=>\mathrm{C} 2 \mathrm{H}+\mathrm{CH} 2 \mathrm{CO}$ ', [1.500000e+12, 0.0, 38000.0])

reaction ('C6H10O5 $=>\mathrm{C} 6 \mathrm{H} 6 \mathrm{O} 3+2 \mathrm{H} 2 \mathrm{O}$ ', [1.000000e+14, 0.0, 65000.0])

reaction $(' \mathrm{C} 6 \mathrm{H} 10 \mathrm{O} 5=>\mathrm{C} 6 \mathrm{H} 8 \mathrm{O} 4+\mathrm{H} 2 \mathrm{O}$ ', [1.000000e+14, 0.0, 65000.0])

reaction('C6H8O4 $=>\mathrm{C} 6 \mathrm{H} 6 \mathrm{O} 3+\mathrm{H} 2 \mathrm{O}$ ', [1.000000e+14, 0.0, 65000.0])

reaction $\left(' \mathrm{C} 5 \mathrm{H} 8 \mathrm{O} 4=>\mathrm{C} 2 \mathrm{H} 4 \mathrm{O} 2+\mathrm{CH} 2 \mathrm{CHO}+\mathrm{HCO}^{\prime},[1.700000 \mathrm{e}+16,0.0,82000.0]\right)$

reaction $(' \mathrm{C} 5 \mathrm{H} 8 \mathrm{O} 4=>\mathrm{C} 5 \mathrm{H} 4 \mathrm{O} 2+2 \mathrm{H} 2 \mathrm{O}$ ', [1.000000e+14, 0.0, 65000.0])

reaction $(' \mathrm{C} 9 \mathrm{H} 10 \mathrm{O} 2=>0.5 \mathrm{C} 10 \mathrm{H} 8+2 \mathrm{CH} 2 \mathrm{CHO}$ ', [3.300000e+15, 0.0, 72000.0])

reaction $\left(' \mathrm{C} 9 \mathrm{H} 10 \mathrm{O} 2+\mathrm{H}=>0.5 \mathrm{C} 2 \mathrm{H} 4+0.5 \mathrm{C} 2 \mathrm{H} 5+\mathrm{C} 6 \mathrm{H} 5 \mathrm{OH}+0.5 \mathrm{CO}+0.5 \mathrm{HCO}^{\prime},[1.000000 \mathrm{e}+13,0.0,5000.0]\right)$

reaction('C11H12O4 + H => C2 $\mathrm{H} 3+\mathrm{C} 8 \mathrm{H} 10 \mathrm{O} 3+\mathrm{CO}$ ', [1.000000e+13, 0.0, 5000.0])

reaction(' $\mathrm{C} 3 \mathrm{H} 3+\mathrm{OH} \Leftrightarrow=>\mathrm{C} 2 \mathrm{H} 3 \mathrm{CHO}$ ', [3.000000e+13, 0.0, 0.0])

reaction $\left(' \mathrm{C} 2 \mathrm{H} 3 \mathrm{CHO}+\mathrm{OH}=>\mathrm{CH} 3 \mathrm{CHO}+\mathrm{HCO}^{\prime},[4.000000 \mathrm{e}+12,0.0,0.0]\right)$

reaction $(' \mathrm{C} 2 \mathrm{H} 3 \mathrm{CHO}+\mathrm{OH}=>\mathrm{C} 2 \mathrm{H} 4+\mathrm{CO} 2+\mathrm{H}$ ', [1.100000e+13, 0.0, 0.0])

reaction $(' \mathrm{CHCHCH} 3+\mathrm{O} 2=>0.2 \mathrm{C} 2 \mathrm{H} 3 \mathrm{CHO}+0.4 \mathrm{CH} 2 \mathrm{O}+0.4 \mathrm{CH} 3 \mathrm{CHO}+0.4 \mathrm{CH} 3 \mathrm{CO}+0.4 \mathrm{HCO}+0.2 \mathrm{OH}$ ', [6.000000e+12,

$0.0,0.0])$

reaction(' $\mathrm{C} 2 \mathrm{H} 3 \mathrm{CHO}+\mathrm{H}=>\mathrm{C} 2 \mathrm{H} 3+\mathrm{CH} 2 \mathrm{O}$ ', [3.000000e+13, 0.0, 5000.0])

reaction ('C2 $\left.\mathrm{H} 3 \mathrm{CHO}+\mathrm{H}=>\mathrm{C} 2 \mathrm{H} 4+\mathrm{HCO}^{\prime},[1.000000 \mathrm{e}+13,0.0,5000.0]\right)$

reaction('C6H5OCH3 $<=>$ C6H5O + CH3', [1.500000e+15, 0.0, 63000.0])

falloff_reaction('C6H5O $+\mathrm{H}(+\mathrm{M})<=>\mathrm{C} 6 \mathrm{H} 5 \mathrm{OH}(+\mathrm{M})$ ',

$\mathrm{kf}=[4.000000 \mathrm{e}+14,0.0,0.0]$,

$\mathrm{kf0}=[3.000000 \mathrm{e}+20,0.0,0.0]$,

efficiencies='AR:0.7 C2H6:3.0 CH4:2.0 CO:1.5 CO2:2.0 H2:2.0 H2O:6.0 ',

falloff $=\operatorname{Troe}(\mathrm{A}=0.2, \mathrm{~T} 3=1 \mathrm{e}-30, \mathrm{~T} 1=1.0000000000000002 \mathrm{e}+30, \mathrm{~T} 2=1 \mathrm{e}-10))$

reaction('C6H5OH $<=>\mathrm{CO}+\mathrm{CYC} 5 \mathrm{H} 6$ ', [2.500000e+13, 0.0, 72400.0])

reaction $\left(' \mathrm{C} 6 \mathrm{H} 5 \mathrm{OH}+\mathrm{O} 2=>\mathrm{C} 2 \mathrm{H} 2+\mathrm{CH} 2 \mathrm{CO}+\mathrm{CO}+\mathrm{H}+\mathrm{HCO}^{\prime},[1.000000 \mathrm{e}+17,0.0,53000.0]\right)$

reaction(' $\left.\mathrm{C} 6 \mathrm{H} 5 \mathrm{OH}+\mathrm{O} 2=>\mathrm{C} 4 \mathrm{H} 6+\mathrm{CO}+\mathrm{CO}^{\prime},[2.000000 \mathrm{e}+17,0.0,55500.0]\right)$

reaction('C6H5OH $\left.+\mathrm{H}<=>\mathrm{C} 6 \mathrm{H} 6+\mathrm{OH}^{\prime},[1.200000 \mathrm{e}+13,0.0,6000.0]\right)$

reaction $\left(' \mathrm{C} 6 \mathrm{H} 5 \mathrm{OH}+\mathrm{OH}=>\mathrm{CO}+\mathrm{CYC} 5 \mathrm{H} 6+\mathrm{OH}^{\prime},[4.000000 \mathrm{e}+12,0.0,0.0]\right)$

reaction('C6H5O => CO + CYC5H5', [2.000000e+11, 0.0, 43920.0]) 
reaction('C6H5O + H $<=>\mathrm{CO}+\mathrm{CYC} 5 \mathrm{H} 6$ ', [2.000000e $+14,0.0,0.0])$

reaction('C6H5O + O $\Rightarrow \mathrm{C} 4 \mathrm{H} 5+2 \mathrm{CO}$ ', [1.000000e+14, 0.0, 0.0])

reaction('C6H5 + O2 $\Leftrightarrow=>$ C6H5O + O', [2.600000e+13, 0.0, 6120.0])

reaction('C6H6 + O $=>$ C6H5O + H', [1.500000e+13, 0.0, 4000.0])

reaction('C7H8 $\Leftrightarrow=>\mathrm{C} 7 \mathrm{H} 7+\mathrm{H}$ ', [4.500000e+15, 0.0, 89200.0])

reaction $\left(' \mathrm{C} 7 \mathrm{H} 7+\mathrm{O} 2=>\mathrm{C} 6 \mathrm{H} 5+\mathrm{CH} 2 \mathrm{O}+\mathrm{O}^{\prime},[6.000000 \mathrm{e}+11,0.0,14500.0]\right)$

reaction('C7H8 + H $\Leftrightarrow=>$ C6H6 + CH3', [1.200000e+13, 0.0, 5100.0])

reaction('C7H8 + CH3 $<=>$ C7H7 + CH4', [4.000000e-05, 5.62, 9000.0])

reaction('C7H7 $\Leftrightarrow=>\mathrm{C} 2 \mathrm{H} 2+\mathrm{CYC} 5 \mathrm{H} 5$ ', [5.000000e+13, 0.0, 70000.0])

reaction ('C6H5O + CH3 $=>$ CRESOL', [3.300000e+12, 0.0, 0.0])

reaction('CRESOL $+\mathrm{H}<=>\mathrm{C} 6 \mathrm{H} 5 \mathrm{OH}+\mathrm{CH} 3$ ', [5.000000e+13, 0.0, 5000.0])

reaction('RCRESOLC $<=>$ RCRESOLO', [1.000000e+11, 0.0, 30000.0])

reaction('HCOOH $\Leftrightarrow=>\mathrm{CO}+\mathrm{H}_{2} \mathrm{O}$ ', [9.000000e+13, 0.0, 65300.0])

reaction('C3H6O2 $\Leftrightarrow=>\mathrm{C} 2 \mathrm{H} 3 \mathrm{CHO}+\mathrm{H}_{2} \mathrm{O}$ ', [4.000000e+13, 0.0, 51000.0])

reaction('C3H6O2 $\Leftrightarrow>\mathrm{CH} 2 \mathrm{O}+\mathrm{CH} 3 \mathrm{CHO}$ ', [4.000000e+13, 0.0, 54000.0])

reaction ('CH2CO + OH $=>\mathrm{H} 2 \mathrm{O}+\mathrm{HCCO}^{\prime}$, [1.198000e+06, 2.0, -3529.84])

reaction $\left(' \mathrm{CH} 2 \mathrm{CO}+\mathrm{O}=>\mathrm{HCCO}+\mathrm{OH}^{\prime},[4.060000 \mathrm{e}+06,2.0,1356.53]\right)$

reaction $(' \mathrm{CH} 4+\mathrm{OH}=>\mathrm{CH} 3+\mathrm{H} 2 \mathrm{O}$ ', [2.796000e+06, 2.0, 1566.11])

reaction(' $\left.\mathrm{C} 2 \mathrm{H} 2+\mathrm{O}=>\mathrm{C} 2 \mathrm{H}+\mathrm{OH}^{\prime},[2.707000 \mathrm{e}+06,2.0,8781.96]\right)$

reaction (' $\mathrm{C} 2 \mathrm{H} 4+\mathrm{H}=>\mathrm{C} 2 \mathrm{H} 3+\mathrm{H} 2$ ', [1.925000e+07, 2.0, 10409.77])

reaction(' $\left.\mathrm{C} 2 \mathrm{H} 4+\mathrm{O}=>\mathrm{C} 2 \mathrm{H} 3+\mathrm{OH}^{\prime},[1.083000 \mathrm{e}+07,2.0,8781.96]\right)$

reaction ('C2 $\mathrm{H} 4+\mathrm{CH} 3=>\mathrm{C} 2 \mathrm{H} 3+\mathrm{CH} 4$ ', [3.122000e+05, 2.0, 11393.6])

reaction(' $\mathrm{C} 2 \mathrm{H} 6+\mathrm{OH}=>\mathrm{C} 2 \mathrm{H} 5+\mathrm{H} 2 \mathrm{O}$ ', [3.595000e+06, 2.0, -238.2])

reaction(' $\left.\mathrm{C} 2 \mathrm{H} 6+\mathrm{O}=>\mathrm{C} 2 \mathrm{H} 5+\mathrm{OH}^{\prime},[1.218000 \mathrm{e}+07,2.0,5025.57]\right)$

reaction $\left(' \mathrm{C} 3 \mathrm{H} 6+\mathrm{O}=>\mathrm{CH} 2 \mathrm{CHCH} 2+\mathrm{OH}^{\prime},[4.060000 \mathrm{e}+06,2.0,2579.54]\right)$

reaction('C3H6 + H => CHCHCH3 + H2', [1.053000e+07, 2.0, 10122.65])

reaction (' $\mathrm{C} 3 \mathrm{H} 6+\mathrm{OH}=>\mathrm{CHCHCH} 3+\mathrm{H} 2 \mathrm{O}$ ', [1.747000e+06, 2.0, 1484.61])

reaction (' $\mathrm{C} 4 \mathrm{H} 4+\mathrm{H}=>\mathrm{C} 4 \mathrm{H} 3+\mathrm{H} 2$ ', [3.369000e+07, 2.0, 10152.16])

reaction $(' \mathrm{C} 4 \mathrm{H} 4+\mathrm{OH}=>\mathrm{C} 4 \mathrm{H} 3+\mathrm{H} 2 \mathrm{O}$ ', [5.592000e+06, 2.0, 1698.84])

reaction('C4H6 + H => C4H5 + H2', [5.776000e+07, 2.0, 10398.54])

reaction $(' \mathrm{C} 4 \mathrm{H} 6+\mathrm{OH}=>\mathrm{C} 4 \mathrm{H} 5+\mathrm{H} 2 \mathrm{O}$ ', [9.586000e+06, 2.0, 1675.91])

reaction $(' \mathrm{H}+\mathrm{NC} 4 \mathrm{H} 8=>\mathrm{H} 2+\mathrm{SC} 4 \mathrm{H} 7$ ', [1.203000e+07, 2.0, 4202.36])

reaction('CH3 + $\mathrm{H} 2 \mathrm{O}=>\mathrm{CH} 4+\mathrm{OH}$ ', [3.903000e+05, 2.0, 15366.11])

reaction $(' \mathrm{H} 2 \mathrm{O} 2+\mathrm{OH}=>\mathrm{H} 2 \mathrm{O}+\mathrm{HO} 2$ ', [3.195000e+05, 2.0, -4169.95])

reaction $(' \mathrm{CH} 3 \mathrm{OH}+\mathrm{OH}=>\mathrm{CH} 3 \mathrm{O}+\mathrm{H} 2 \mathrm{O}$ ', [1.997000e+05, 2.0, -2715.03])

reaction $\left(' \mathrm{CH} 2 \mathrm{O}+\mathrm{OH}=>\mathrm{H} 2 \mathrm{O}+\mathrm{HCO}^{\prime},[3.195000 \mathrm{e}+06,2.0,-2065.87]\right)$

reaction $\left(' \mathrm{CH} 2 \mathrm{O}+\mathrm{O}=>\mathrm{HCO}+\mathrm{OH}^{\prime},[1.083000 \mathrm{e}+07,2.0,1094.46]\right)$

reaction $\left(' \mathrm{CH} 2 \mathrm{O}+\mathrm{CH} 3=>\mathrm{CH} 4+\mathrm{HCO}^{\prime},[3.122000 \mathrm{e}+05,2.0,3781.38]\right)$

reaction(' $\mathrm{CH} 3 \mathrm{CHO}+\mathrm{OH}=>\mathrm{CH} 3 \mathrm{CO}+\mathrm{H} 2 \mathrm{O}$ ', [2.396000e+06, 2.0, -1734.99])

reaction ('H + MCPTD $=>\mathrm{C} 6 \mathrm{H} 6+\mathrm{H}+\mathrm{H} 2$ ', [2.407000e $+07,2.0,2663.07])$

reaction('C6H6 + O $=>\mathrm{C} 6 \mathrm{H} 5+\mathrm{OH}$ ', [2.165000e+07, 2.0, 8781.96])

reaction $\left(' \mathrm{C} 2 \mathrm{H} 5 \mathrm{OH}+\mathrm{OH}=>\mathrm{C} 2 \mathrm{H} 4 \mathrm{OH}+\mathrm{H}_{2} \mathrm{O}\right.$ ', [1.198000e+06, 2.0, -474.43])

reaction(' $\mathrm{C} 2 \mathrm{H} 5 \mathrm{OH}+\mathrm{H}=>\mathrm{CH} 3 \mathrm{CHOH}+\mathrm{H2}$ ', [6.257000e+06, 2.0, 3950.57]) 
reaction $\left(' \mathrm{C} 2 \mathrm{H} 5 \mathrm{OH}+\mathrm{OH}=>\mathrm{CH} 3 \mathrm{CHOH}+\mathrm{H}_{2} \mathrm{O}\right.$ ', [1.038000e+06, 2.0, -2259.83])

reaction(' $\mathrm{C} 2 \mathrm{H} 4 \mathrm{O} 2+\mathrm{H}=>\mathrm{CH} 2 \mathrm{OH}+\mathrm{CO}+\mathrm{H} 2$ ', [9.627000e+06, 2.0, 2387.18])

reaction $\left(' \mathrm{C} 2 \mathrm{H} 4 \mathrm{O} 2+\mathrm{OH}=>\mathrm{CH} 2 \mathrm{OH}+\mathrm{CO}+\mathrm{H}_{2} \mathrm{O}\right.$ ', [1.598000e+06, 2.0, -3343.83])

reaction $\left(' \mathrm{C} 2 \mathrm{H} 4 \mathrm{O} 2+\mathrm{CH} 3=>\mathrm{CH} 2 \mathrm{OH}+\mathrm{CH} 4+\mathrm{CO}^{\prime},[1.561000 \mathrm{e}+05,2.0,3259.77]\right)$

reaction(' $\mathrm{C} 2 \mathrm{H} 4 \mathrm{O} 2+\mathrm{H}=>\mathrm{C} 2 \mathrm{H} 2 \mathrm{O} 2+\mathrm{H}+\mathrm{H} 2$ ', [2.407000e+06, 2.0, 3950.57])

reaction $(' \mathrm{C} 2 \mathrm{H} 4 \mathrm{O} 2+\mathrm{OH}=>\mathrm{C} 2 \mathrm{H} 2 \mathrm{O} 2+\mathrm{H}+\mathrm{H} 2 \mathrm{O}$ ', [3.994000e+05, 2.0, -2259.83])

reaction $\left(' \mathrm{C} 2 \mathrm{H} 2 \mathrm{O} 2+\mathrm{H}=>\mathrm{CO}+\mathrm{H} 2+\mathrm{HCO}^{\prime},[1.925000 \mathrm{e}+07,2.0,2387.18]\right)$

reaction $\left(' \mathrm{C} 2 \mathrm{H} 2 \mathrm{O} 2+\mathrm{OH}=>\mathrm{CO}+\mathrm{H} 2 \mathrm{O}+\mathrm{HCO}^{\prime}\right.$, [3.195000e+06, 2.0, -3343.83])

reaction $\left(' \mathrm{C} 2 \mathrm{H} 2 \mathrm{O} 2+\mathrm{O}=>\mathrm{CO}+\mathrm{HCO}+\mathrm{OH}^{\prime},[1.083000 \mathrm{e}+07,2.0,1094.46]\right)$

reaction $\left(' \mathrm{C} 2 \mathrm{H} 2 \mathrm{O} 2+\mathrm{CH} 3=>\mathrm{CH} 4+\mathrm{CO}+\mathrm{HCO}^{\prime}\right.$, [3.122000e+05, 2.0, 3259.77])

reaction (' $\mathrm{C} 3 \mathrm{H} 4 \mathrm{O} 3+\mathrm{H}=>\mathrm{C} 2 \mathrm{H} 2 \mathrm{O} 2+\mathrm{CO}+\mathrm{H}+\mathrm{H} 2$ ', [1.925000e+07, 2.0, 2387.18])

reaction $\left(' \mathrm{C} 3 \mathrm{H} 4 \mathrm{O} 3+\mathrm{OH}=>\mathrm{C} 2 \mathrm{H} 2 \mathrm{O} 2+\mathrm{CO}+\mathrm{H}+\mathrm{H}_{2} \mathrm{O}\right.$ ', [3.195000e+06, 2.0, -3343.83])

reaction(' $\mathrm{C} 6 \mathrm{H} 6 \mathrm{O} 3+\mathrm{H}=>\mathrm{C} 5 \mathrm{H} 4 \mathrm{O} 2+\mathrm{CO}+\mathrm{H}+\mathrm{H} 2$ ', [9.627000e+06, 2.0, 2387.18])

reaction $\left(' \mathrm{C} 6 \mathrm{H} 6 \mathrm{O} 3+\mathrm{OH}=>\mathrm{C} 5 \mathrm{H} 4 \mathrm{O} 2+\mathrm{CO}+\mathrm{H}+\mathrm{H}_{2} \mathrm{O}\right.$ ', [1.598000e+06, 2.0, -3343.83])

reaction (' $\mathrm{C} 5 \mathrm{H} 4 \mathrm{O} 2+\mathrm{H}=>\mathrm{C} 3 \mathrm{H} 3+2 \mathrm{CO}+\mathrm{H} 2$ ', [9.627000e+06, 2.0, 2387.18])

reaction $\left(' \mathrm{C} 5 \mathrm{H} 4 \mathrm{O} 2+\mathrm{OH}=>\mathrm{C} 3 \mathrm{H} 3+2 \mathrm{CO}+\mathrm{H}_{2} \mathrm{O}\right.$ ', [1.598000e+06, 2.0, -3343.83])

reaction (' $\mathrm{C} 5 \mathrm{H} 4 \mathrm{O} 2+\mathrm{H}=>\mathrm{C} 4 \mathrm{H} 3 \mathrm{O}+\mathrm{CO}+\mathrm{H} 2$ ', [9.627000e+06, 2.0, 2387.18])

reaction $\left(' \mathrm{C} 5 \mathrm{H} 4 \mathrm{O} 2+\mathrm{OH}=>\mathrm{C} 4 \mathrm{H} 3 \mathrm{O}+\mathrm{CO}+\mathrm{H}_{2} \mathrm{O} ',[1.598000 \mathrm{e}+06,2.0,-3343.83]\right)$

reaction $\left(' \mathrm{C} 5 \mathrm{H} 4 \mathrm{O} 2+\mathrm{O}=>\mathrm{C} 4 \mathrm{H} 3 \mathrm{O}+\mathrm{CO}+\mathrm{OH}^{\prime}\right.$, [5.413000e+06, 2.0, 1094.46])

reaction('C6H10O5 + $\mathrm{H}=>\mathrm{C} 3 \mathrm{H} 4 \mathrm{O} 3+\mathrm{CH} 2 \mathrm{CHO}+\mathrm{CH} 2 \mathrm{O}+\mathrm{H} 2$ ', [9.627000e+06, 2.0, 3950.57])

reaction('C6H10O5 $+\mathrm{OH}=>\mathrm{C} 3 \mathrm{H} 4 \mathrm{O} 3+\mathrm{CH} 2 \mathrm{CHO}+\mathrm{CH} 2 \mathrm{O}+\mathrm{H}_{2} \mathrm{O}$ ', [1.598000e+06, 2.0, -2259.83])

reaction('C6H10O5 + H => C6H8O4 + $\left.\mathrm{H} 2+\mathrm{OH}^{\prime},[1.203000 \mathrm{e}+06,2.0,3950.57]\right)$

reaction('C6H10O5 + OH $=>$ C6H8O4 $\left.+\mathrm{H} 2 \mathrm{O}+\mathrm{OH}^{\prime},[1.997000 \mathrm{e}+05,2.0,-2259.83]\right)$

reaction ('C5H8O4 + $\mathrm{H}=>\mathrm{C} 5 \mathrm{H} 4 \mathrm{O} 2+\mathrm{H} 2+\mathrm{H} 2 \mathrm{O}+\mathrm{OH}^{\prime}$, [4.813000e+06, 2.0, 3950.57])

reaction $\left(' \mathrm{C} 5 \mathrm{H} 8 \mathrm{O} 4+\mathrm{OH}=>\mathrm{C} 5 \mathrm{H} 4 \mathrm{O} 2+2 \mathrm{H} 2 \mathrm{O}+\mathrm{OH}^{\prime},[7.988000 \mathrm{e}+05,2.0,-2259.83]\right)$

reaction('C5H8O4 + $\mathrm{H}=>\mathrm{C} 2 \mathrm{H} 4 \mathrm{O} 2+\mathrm{CH} 2 \mathrm{CHO}+\mathrm{CO}+\mathrm{H} 2$ ', [4.813000e+06, 2.0, 3950.57])

reaction(' $\mathrm{C} 5 \mathrm{H} 8 \mathrm{O} 4+\mathrm{OH}=>\mathrm{C} 2 \mathrm{H} 4 \mathrm{O} 2+\mathrm{CH} 2 \mathrm{CHO}+\mathrm{CO}+\mathrm{H}_{2} \mathrm{O}$ ', [7.988000e+05, 2.0, -2259.83])

reaction (' $\mathrm{C} 5 \mathrm{H} 8 \mathrm{O} 4+\mathrm{H}=>\mathrm{C} 2 \mathrm{H} 2 \mathrm{O} 2+0.5 \mathrm{C} 2 \mathrm{H} 3 \mathrm{CHO}+0.5 \mathrm{CH} 3 \mathrm{CHO}+\mathrm{H} 2+0.5 \mathrm{HCO}+0.5 \mathrm{OH}$ ', [4.813000e+06, 2.0, 3950.57])

reaction $(' \mathrm{C} 5 \mathrm{H} 8 \mathrm{O} 4+\mathrm{OH}=>\mathrm{C} 2 \mathrm{H} 2 \mathrm{O} 2+0.5 \mathrm{C} 2 \mathrm{H} 3 \mathrm{CHO}+0.5 \mathrm{CH} 3 \mathrm{CHO}+\mathrm{H} 2 \mathrm{O}+0.5 \mathrm{HCO}+0.5 \mathrm{OH}$ ', [7.988000e+05, 2.0, 2259.83])

reaction('C5H8O4 + O $=>\mathrm{C} 2 \mathrm{H} 2 \mathrm{O} 2+0.5 \mathrm{C} 2 \mathrm{H} 3 \mathrm{CHO}+0.5 \mathrm{CH} 3 \mathrm{CHO}+0.5 \mathrm{HCO}+1.5 \mathrm{OH}$ ', [2.707000e+06, 2.0, 2579.54])

reaction $(' \mathrm{C} 9 \mathrm{H} 10 \mathrm{O} 2+\mathrm{H}=>0.5 \mathrm{C} 10 \mathrm{H} 8+\mathrm{CH} 2 \mathrm{CHO}+\mathrm{CH} 2 \mathrm{CO}+\mathrm{H} 2 ',[9.627000 \mathrm{e}+06,2.0,2387.18])$

reaction $\left(' \mathrm{C} 9 \mathrm{H} 10 \mathrm{O} 2+\mathrm{OH}=>0.5 \mathrm{C} 10 \mathrm{H} 8+\mathrm{CH} 2 \mathrm{CHO}+\mathrm{CH} 2 \mathrm{CO}+\mathrm{H}_{2} \mathrm{O}\right.$ ', [1.598000e+06, 2.0, -3343.83])

reaction ('C8H10O3 $+\mathrm{H}=>0.5 \mathrm{AC} 3 \mathrm{H} 4+\mathrm{C} 2 \mathrm{H} 2+\mathrm{CH} 3 \mathrm{O}+2 \mathrm{CO}+\mathrm{H} 2+0.5 \mathrm{PC} 3 \mathrm{H} 4$ ', [1.203000e+07, 2.0, 6525.57])

reaction $(' \mathrm{C} 8 \mathrm{H} 10 \mathrm{O} 3+\mathrm{H}=>\mathrm{C} 2 \mathrm{H} 2+0.5 \mathrm{C} 4 \mathrm{H} 4+\mathrm{CH} 2 \mathrm{CO}+\mathrm{CH} 3 \mathrm{O}+\mathrm{CO}+\mathrm{H} 2$ ', [1.203000e+07, 2.0, 6525.57])

reaction $\left(' \mathrm{C} 2 \mathrm{H} 3 \mathrm{CHO}+\mathrm{H}=>0.1 \mathrm{C} 2 \mathrm{H} 2+0.9 \mathrm{C} 2 \mathrm{H} 3+0.9 \mathrm{CO}+\mathrm{H} 2+0.1 \mathrm{HCO}^{\prime},[1.444000 \mathrm{e}+07,2.0,2387.18]\right)$

reaction $\left(' \mathrm{C} 2 \mathrm{H} 3 \mathrm{CHO}+\mathrm{OH}=>0.1 \mathrm{C} 2 \mathrm{H} 2+0.9 \mathrm{C} 2 \mathrm{H} 3+0.9 \mathrm{CO}+\mathrm{H} 2 \mathrm{O}+0.1 \mathrm{HCO}^{\prime}\right.$, [2.396000e+06, 2.0, -3343.83])

reaction ('C6H5OH $+\mathrm{H}=>\mathrm{C} 6 \mathrm{H} 5 \mathrm{O}+\mathrm{H} 2$ ', [1.203000e+07, 2.0, 6706.81])

reaction $(' \mathrm{C} 6 \mathrm{H} 5 \mathrm{OH}+\mathrm{OH}=>\mathrm{C} 6 \mathrm{H} 5 \mathrm{O}+\mathrm{H} 2 \mathrm{O}$ ', [1.997000e+06, 2.0, 841.27])

reaction(' $\mathrm{C} 6 \mathrm{H} 5 \mathrm{OH}+\mathrm{O}=>\mathrm{C} 6 \mathrm{H} 5 \mathrm{O}+\mathrm{OH}$ ', [6.767000e+06, 2.0, 5025.57])

reaction('CRESOL + H => H2 + 0.75 RCRESOLC + 0.25 RCRESOLO', [1.925000e+07, 2.0, 3950.57])

reaction ('CH3COOH $\left.+\mathrm{H}=>\mathrm{CH} 2 \mathrm{CO}+\mathrm{H} 2+\mathrm{OH}^{\prime},[7.220000 \mathrm{e}+06,2.0,6525.57]\right)$

reaction $\left(' \mathrm{CH} 3 \mathrm{COOH}+\mathrm{OH}=>\mathrm{CH} 2 \mathrm{CO}+\mathrm{H} 2 \mathrm{O}+\mathrm{OH}^{\prime},[1.198000 \mathrm{e}+06,2.0,-474.43]\right)$ 
reaction(' $\mathrm{CH} 3 \mathrm{COOH}+\mathrm{H}=>\mathrm{CH} 3+\mathrm{CO} 2+\mathrm{H} 2$ ', [2.407000e+06, 2.0, 6525.57])

reaction $(' \mathrm{CH} 3 \mathrm{COOH}+\mathrm{OH}=>\mathrm{CH} 3+\mathrm{CO} 2+\mathrm{H} 2 \mathrm{O}$ ', [3.994000e+05, 2.0, -474.43])

three_body_reaction('H $+\mathrm{OH}+\mathrm{M}<=>\mathrm{H} 2 \mathrm{O}+\mathrm{M}^{\prime},[4.500000 \mathrm{e}+22,-2.0,0.0]$,

efficiencies='CO2:1.9 H2:2.0 H2O:16.0 ')

falloff_reaction('C2H3 + H $(+\mathrm{M})<=>\mathrm{C} 2 \mathrm{H} 4(+\mathrm{M})$ ',

$\mathrm{kf}=[6.080000 \mathrm{e}+12,0.27,280.0]$,

$\mathrm{kf0}=[1.400000 \mathrm{e}+30,-3.86,3320.0]$,

efficiencies='AR:0.7 C2H6:3.0 CH4:2.0 CO:1.5 CO2:2.0 H2:2.0 H2O:6.0 ',

falloff $=\operatorname{Troe}(\mathrm{A}=0.782, \mathrm{~T} 3=207.49999999999997, \mathrm{~T} 1=2663.0, \mathrm{~T} 2=6095.0))$

reaction('CHCHCH3 $<=>\mathrm{C} 2 \mathrm{H} 2+\mathrm{CH} 3$ ', [2.000000e+13, 0.0, 38000.0])

reaction(' $\mathrm{C} 2 \mathrm{H} 3+\mathrm{CH} 3<=>\mathrm{CH} 2 \mathrm{CHCH} 2+\mathrm{H}$ ', [5.000000e+01, 3.7, 5677.0])

falloff_reaction ('CH3OH $(+\mathrm{M})<=>\mathrm{CH} 2 \mathrm{OH}+\mathrm{H}(+\mathrm{M})$ ',

$\mathrm{kf}=[1.540000 \mathrm{e}+16,0.0,96800.0]$,

$\mathrm{kf}=[7.200000 \mathrm{e}+46,-7.93,107700.0])$

reaction('C4H2 $\left.+\mathrm{OH}<=>\mathrm{C} 2 \mathrm{H} 2+\mathrm{HCCO}^{\prime},[5.000000 \mathrm{e}+12,0.0,0.0]\right)$

reaction $\left(' \mathrm{CH} 2 \mathrm{CO}+\mathrm{O} 2=>\mathrm{CO}+\mathrm{HCO}+\mathrm{OH}^{\prime},[3.000000 \mathrm{e}+14,0.0,40000.0]\right)$

reaction('CH2OH $+\mathrm{H}=>\mathrm{CH} 2 \mathrm{O}+\mathrm{H} 2$ ', [2.000000e+13, 0.0, 0.0])

reaction('CH2CHO + H => CH3CHO', [6.000000e+13, 0.0, 0.0])

reaction('CYC5H6 + O2 => CH2CHCH2 + CO + HCO', [8.000000e+13, 0.0, 39000.0])

reaction $(' \mathrm{C} 8 \mathrm{H} 10 \mathrm{O} 3+\mathrm{H}=>0.5 \mathrm{C} 6 \mathrm{H} 5 \mathrm{OH}+0.5 \mathrm{C} 8 \mathrm{H} 10 \mathrm{O} 3+\mathrm{CH} 3 \mathrm{O}$ ', [1.000000e+13, 0.0, 5000.0])

reaction $(' \mathrm{CH} 3 \mathrm{COOH}<=>\mathrm{CH} 4+\mathrm{CO} 2$ ', [1.500000e+13, 0.0, 70000.0])

reaction $\left(' \mathrm{C} 2 \mathrm{H} 3+\mathrm{H} 2=>\mathrm{C} 2 \mathrm{H} 4+\mathrm{H}^{\prime},[9.496000 \mathrm{e}+05,2.0,8459.77]\right)$

reaction $\left(' \mathrm{C} 7 \mathrm{H} 7+\mathrm{H} 2=>\mathrm{C} 7 \mathrm{H} 8+\mathrm{H}^{\prime}\right.$, [3.780000e+05, 2.0, 19839.23])

reaction('C3H8 + OH => H2O + NC3H7', [3.195000e+06, 2.0, -498.69])

reaction $(' \mathrm{CH} 3 \mathrm{CHO}+\mathrm{HO} 2=>\mathrm{CH} 3 \mathrm{CO}+\mathrm{H} 2 \mathrm{O} 2$ ', [3.233000e+05, 2.0, 8726.73])

reaction(' $\left.\mathrm{C} 2 \mathrm{H} 4 \mathrm{O} 2+\mathrm{O}=>\mathrm{CH} 2 \mathrm{OH}+\mathrm{CO}+\mathrm{OH}^{\prime},[5.413000 \mathrm{e}+06,2.0,1094.46]\right)$

reaction('C2H4O2 + $\mathrm{HO} 2=>\mathrm{CH} 2 \mathrm{OH}+\mathrm{CO}+\mathrm{H} 2 \mathrm{O} 2$ ', [2.155000e+05, 2.0, 9982.61])

reaction('C6H6O3 + $\left.\mathrm{CH} 3=>\mathrm{C} 5 \mathrm{H} 4 \mathrm{O} 2+\mathrm{CH} 4+\mathrm{CO}+\mathrm{H}^{\prime},[1.561000 \mathrm{e}+05,2.0,3259.77]\right)$

reaction('C5H4O2 + $\mathrm{O}=>\mathrm{C} 3 \mathrm{H} 3+2 \mathrm{CO}+\mathrm{OH}^{\prime}$, [5.413000e+06, 2.0, 1094.46])

reaction('C5H4O2 + CH3 => C3H3 + CH4 + 2 CO', [1.561000e+05, 2.0, 3259.77])

reaction('C5H8O4 + $\mathrm{O}=>\mathrm{C} 5 \mathrm{H} 4 \mathrm{O} 2+\mathrm{H} 2 \mathrm{O}+2 \mathrm{OH}$, [2.707000e+06, 2.0, 2579.54])

three_body_reaction('H2 $+\mathrm{M} \Leftrightarrow=>2 \mathrm{H}+\mathrm{M}^{\prime}$, [1.115000e+14, 0.0, 96081.0],

efficiencies='AR:0.5 CO:1.9 CO2:3.8 H2:2.5 H2O:12.0 ')

reaction('C3H3 $\left.<=>\mathrm{C} 3 \mathrm{H} 2+\mathrm{H}^{\prime},[1.000000 \mathrm{e}+13,0.0,79000.0]\right)$

reaction('2 C2H2 <=> C4H4', [1.500000e+12, 0.0, 37400.0])

reaction('C4H4 $\Leftrightarrow=>\mathrm{C} 4 \mathrm{H} 2+\mathrm{H} 2$ ', [3.500000e+11, 0.0, 66000.0])

reaction(' $\left.\mathrm{C} 2 \mathrm{H} 3+\mathrm{O}=>\mathrm{CH} 2 \mathrm{CHO}^{\prime},[2.500000 \mathrm{e}+13,0.0,0.0]\right)$

reaction('HCO $\left.+\mathrm{O}<=>\mathrm{CO} 2+\mathrm{H}^{\prime},[3.000000 \mathrm{e}+13,0.0,0.0]\right)$

reaction $(' \mathrm{CH} 2 \mathrm{OH}+\mathrm{OH}=>\mathrm{CH} 2 \mathrm{O}+\mathrm{H} 2 \mathrm{O}$ ', [1.500000e+13, 0.0, 0.0])

reaction('CH2 + CH3 $<=>\mathrm{C} 2 \mathrm{H} 4+\mathrm{H}$ ', [4.200000e+13, 0.0, 0.0])

reaction $(' \mathrm{CH} 2+\mathrm{H}<=>\mathrm{CH}+\mathrm{H} 2$ ', [1.750000e+14, 0.0, -165.0])

reaction(' $\left.\mathrm{CH} 2+\mathrm{O}<=>\mathrm{CO}+2 \mathrm{H}^{\prime},[7.000000 \mathrm{e}+13,0.0,0.0]\right)$

reaction $\left(' \mathrm{CH} 2+\mathrm{CO} 2<=>\mathrm{CH} 2 \mathrm{O}+\mathrm{CO}^{\prime},[1.100000 \mathrm{e}+11,0.0,1000.0]\right)$ 
reaction $\left(' \mathrm{CH} 2 \mathrm{~S}+\mathrm{CO} 2 \Leftrightarrow=>\mathrm{CH} 2 \mathrm{O}+\mathrm{CO}^{\prime},[3.000000 \mathrm{e}+12,0.0,0.0]\right)$

reaction(' $\mathrm{CH}+\mathrm{H}<=>\mathrm{C}+\mathrm{H} 2$ ', [1.500000e+14, 0.0, 0.0])

reaction $\left(' \mathrm{CH}+\mathrm{O} 2<=>\mathrm{HCO}+\mathrm{O}^{\prime},[3.300000 \mathrm{e}+13,0.0,0.0]\right)$

reaction(' $\left.\mathrm{CH}+\mathrm{H} 2 \mathrm{O} \Leftrightarrow=>\mathrm{CH} 2 \mathrm{O}+\mathrm{H}^{\prime},[5.700000 \mathrm{e}+12,0.0,-760.0]\right)$

reaction(' $\mathrm{C} 2 \mathrm{H} 3+\mathrm{HCCO}<=>\mathrm{CO}+\mathrm{PC} 3 \mathrm{H} 4$ ', [4.000000e+13, 0.0, 0.0])

reaction(' $\left.\mathrm{C} 3 \mathrm{H} 2+\mathrm{OH}<=>\mathrm{C} 2 \mathrm{H} 2+\mathrm{HCO}^{\prime},[5.000000 \mathrm{e}+13,0.0,0.0]\right)$

reaction('AC3H4 + C2H2 $\Leftrightarrow=>$ CYC5H6', [4.000000e+11, 0.0, 22000.0])

reaction('C2H2 + PC3H4 $<=>$ CYC5H6', [5.000000e+10, 0.0, 22000.0])

reaction $\left(' \mathrm{C} 3 \mathrm{H} 4 \mathrm{O} 3=>\mathrm{C} 2 \mathrm{H} 2 \mathrm{O} 2+\mathrm{H}+\mathrm{HCO}^{\prime},[1.000000 \mathrm{e}+16,0.0,77000.0]\right)$

reaction $\left(' \mathrm{C} 2 \mathrm{H} 3 \mathrm{CHO}=>\mathrm{C} 2 \mathrm{H} 3+\mathrm{HCO}^{\prime},[3.000000 \mathrm{e}+16,0.0,90000.0]\right)$

reaction('C7H8 $\Leftrightarrow=>\mathrm{C} 6 \mathrm{H} 5+\mathrm{CH} 3$ ', [1.200000e+16, 0.0, 99800.0])

reaction(' $\mathrm{CH} 3 \mathrm{COOH} \Leftrightarrow=>\mathrm{CH} 2 \mathrm{CO}+\mathrm{H} 2 \mathrm{O}$ ', [1.400000e+12, 0.0, 65000.0])

reaction (' $\mathrm{C} 3 \mathrm{H} 4 \mathrm{O} 3+\mathrm{O}=>\mathrm{C} 2 \mathrm{H} 2 \mathrm{O} 2+\mathrm{CO}+\mathrm{H}+\mathrm{OH}^{\prime}$, [1.083000e+07, 2.0, 1094.46])

reaction(' $\mathrm{C} 6 \mathrm{H} 6 \mathrm{O} 3+\mathrm{O}=>\mathrm{C} 5 \mathrm{H} 4 \mathrm{O} 2+\mathrm{CO}+\mathrm{H}+\mathrm{OH}^{\prime}$, [5.413000e+06, 2.0, 1094.46])

reaction('C6H8O4 + $\mathrm{H}=>\mathrm{C} 6 \mathrm{H} 6 \mathrm{O} 3+\mathrm{H} 2+\mathrm{OH}^{\prime}$, [2.407000e+06, 2.0, 3950.57])

reaction $\left(' \mathrm{C} 6 \mathrm{H} 8 \mathrm{O} 4+\mathrm{OH}=>\mathrm{C} 6 \mathrm{H} 6 \mathrm{O} 3+\mathrm{H} 2 \mathrm{O}+\mathrm{OH}^{\prime},[3.994000 \mathrm{e}+05,2.0,-2259.83]\right)$

reaction('AC3H4 + OH $\Leftrightarrow=>\mathrm{C} 3 \mathrm{H} 3+\mathrm{H} 2 \mathrm{O}$ ', [2.000000e+07, 2.0, 1000.0])

reaction('C6H10O5 $=>\mathrm{C} 3 \mathrm{H} 4 \mathrm{O} 3+\mathrm{C} 3 \mathrm{H} 6 \mathrm{O} 2$ ', [1.700000e+13, 0.0, 65000.0])

three_body_reaction(' $\mathrm{O}+\mathrm{OH}+\mathrm{M}<=>\mathrm{HO} 2+\mathrm{M}^{\prime}$, [1.000000e+16, 0.0, 0.0])

falloff_reaction('C2H4 $(+\mathrm{M})<=>\mathrm{C} 2 \mathrm{H} 2+\mathrm{H} 2(+\mathrm{M})$ ',

$\mathrm{kf}=[8.000000 \mathrm{e}+12,0.44,88770.0]$,

$\mathrm{kf0}=[1.580000 \mathrm{e}+51,-9.3,97800.0]$,

efficiencies='AR:0.7 C2H6:3.0 CH4:2.0 CO:1.5 CO2:2.0 H2:2.0 H2O:6.0 ',

falloff $=\operatorname{Troe}(\mathrm{A}=0.7345, \mathrm{~T} 3=180.0, \mathrm{~T} 1=1035.0, \mathrm{~T} 2=5417.0))$

reaction('2 $\mathrm{C} 2 \mathrm{H} 2<=>\mathrm{C} 4 \mathrm{H} 3+\mathrm{H}$ ', [2.000000e+16, 0.0, 81500.0])

reaction('C4H3 + H $<=>$ C4H4', [1.000000e+14, 0.0, 0.0])

reaction('2 $\mathrm{C} 3 \mathrm{H} 3=>\mathrm{C} 2 \mathrm{H} 2+\mathrm{C} 4 \mathrm{H} 4$ ', $[1.000000 \mathrm{e}+11,0.0,0.0])$

reaction('AC3H4 <=> PC3H4', [6.026000e+53, -12.18, 84276.0])

reaction('CH3 + CH4 => C2H6 + H', [2.500000e+13, 0.0, 31000.0])

reaction('NC4H8 $<=>\mathrm{C} 4 \mathrm{H} 6+\mathrm{H} 2$ ', [5.000000e+13, 0.0, 70000.0])

reaction $(' \mathrm{AC} 3 \mathrm{H} 4+\mathrm{O} 2=>\mathrm{CH} 2 \mathrm{CO}+\mathrm{CH} 2 \mathrm{O}$ ', [1.000000e+15, 0.0, 41000.0])

reaction(' $\left.\mathrm{C} 2 \mathrm{H} 2+\mathrm{O}=>\mathrm{CH} 2 \mathrm{CO}^{\prime},[1.000000 \mathrm{e}+13,0.0,15000.0]\right)$

reaction('C4H4 + O $\left.\Leftrightarrow=>\mathrm{C} 3 \mathrm{H} 3+\mathrm{HCO}^{\prime},[3.200000 \mathrm{e}+08,1.44,525.0]\right)$

reaction $\left(' \mathrm{C} 4 \mathrm{H} 6+\mathrm{O}=>\mathrm{C} 3 \mathrm{H} 6+\mathrm{CO}^{\prime},[1.500000 \mathrm{e}+12,0.0,0.0]\right)$

reaction(' $\mathrm{CH} 2 \mathrm{O}+\mathrm{OH}=>\mathrm{CO} 2+\mathrm{H}+\mathrm{H} 2$ ', $[1.000000 \mathrm{e}+11,0.0,0.0])$

reaction('AC3H4 $+\mathrm{OH}=>0.5 \mathrm{C} 2 \mathrm{H} 3+0.5 \mathrm{CH} 2 \mathrm{CO}+0.5 \mathrm{CH} 2 \mathrm{O}+0.5 \mathrm{CH} 3$ ', [5.000000e+11, 0.0, 1000.0])

reaction $(' \mathrm{NC} 4 \mathrm{H} 8+\mathrm{OH}=>0.5 \mathrm{C} 2 \mathrm{H} 5+0.5 \mathrm{CH} 2 \mathrm{O}+0.5 \mathrm{CH} 3 \mathrm{CHO}+0.5 \mathrm{NC} 3 \mathrm{H} 7$ ', [1.500000e+12, 0.0, 0.0])

reaction $(' \mathrm{CH} 2 \mathrm{O}+\mathrm{HCO}<=>\mathrm{CH} 3+\mathrm{CO} 2$ ', [5.000000e+11, 0.0, 6000.0])

reaction $\left(' \mathrm{C} 3 \mathrm{H} 6+\mathrm{O} 2=>\mathrm{CH} 2 \mathrm{O}+\mathrm{CH} 3 \mathrm{CHO}^{\prime},[1.000000 \mathrm{e}+14,0.0,39000.0]\right)$

reaction('CH3 + O2 $\left.<=>\mathrm{CH} 3 \mathrm{O}+\mathrm{O}^{\prime},[4.000000 \mathrm{e}+12,0.0,27000.0]\right)$

reaction(' $\left.\mathrm{C} 2 \mathrm{H}+\mathrm{O} 2<=>\mathrm{CO}+\mathrm{HCO}^{\prime},[2.000000 \mathrm{e}+14,0.0,7000.0]\right)$

reaction('C2H5 + $\mathrm{O} 2=>\mathrm{CH} 2 \mathrm{O}+\mathrm{CH} 3 \mathrm{O}$ ', [1.000000e+14, 0.0, 24000.0])

reaction $\left(' \mathrm{CH} 2 \mathrm{CHO}+\mathrm{O} 2=>\mathrm{CH} 2 \mathrm{O}+\mathrm{CO}+\mathrm{OH}^{\prime},[6.000000 \mathrm{e}+10,0.0,0.0]\right)$ 
reaction $\left(' \mathrm{C} 2 \mathrm{H} 3+\mathrm{OH}=>\mathrm{CH} 3 \mathrm{CHO}^{\prime},[5.000000 \mathrm{e}+12,0.0,0.0]\right)$

reaction(' $\mathrm{C} 2 \mathrm{H} 3+\mathrm{OH} \Leftrightarrow=>\mathrm{C} 2 \mathrm{H} 2+\mathrm{H} 2 \mathrm{O}$ ', [4.000000e+12, 0.0, 0.0])

reaction(' $\mathrm{CH} 3+\mathrm{CH} 3 \mathrm{O}=>\mathrm{CH} 2 \mathrm{O}+\mathrm{CH} 4$ ', $[1.000000 \mathrm{e}+13,0.0,0.0])$

reaction(' $\mathrm{CH} 2 \mathrm{CHO}+\mathrm{O} 2=>\mathrm{CH} 2 \mathrm{CO}+\mathrm{HO}_{2}$, [5.000000e+11, 0.0, 3000.0])

three_body_reaction('CH3 $+\mathrm{M}<=>\mathrm{CH} 2+\mathrm{H}+\mathrm{M}^{\prime}$, [1.000000e+16, 0.0, 90600.0])

reaction(' $\mathrm{CH} 2+\mathrm{O}<=>\mathrm{CO}+\mathrm{H} 2$ ', [5.000000e+13, 0.0, 0.0])

reaction $\left(' \mathrm{CH} 2+\mathrm{OH}<=>\mathrm{CH} 2 \mathrm{O}+\mathrm{H}^{\prime},[3.000000 \mathrm{e}+13,0.0,0.0]\right)$

reaction('CH2S + O2 $\left.<=>\mathrm{CO}+\mathrm{H}+\mathrm{OH}^{\prime},[3.100000 \mathrm{e}+13,0.0,0.0]\right)$

reaction(' $\left.\mathrm{CH}+\mathrm{CO} 2<=>\mathrm{CO}+\mathrm{HCO}^{\prime},[3.400000 \mathrm{e}+12,0.0,705.0]\right)$

reaction(' $\left.\mathrm{C} 2 \mathrm{H} 2+\mathrm{CH} 2<=>\mathrm{C} 3 \mathrm{H} 3+\mathrm{H}^{\prime},[1.200000 \mathrm{e}+13,0.0,6600.0]\right)$

reaction(' $\left.\mathrm{C} 2 \mathrm{H} 2+\mathrm{CH} 2 \mathrm{~S}<=>\mathrm{C} 3 \mathrm{H} 3+\mathrm{H}^{\prime},[6.000000 \mathrm{e}+13,0.0,0.0]\right)$

reaction(' $\left.\mathrm{C} 2 \mathrm{H} 2+\mathrm{CH} \Leftrightarrow=>\mathrm{C} 3 \mathrm{H} 2+\mathrm{H}^{\prime},[8.400000 \mathrm{e}+13,0.0,0.0]\right)$

reaction('CH2CO $\left.+\mathrm{CH} 3<=>\mathrm{C} 2 \mathrm{H} 5+\mathrm{CO}^{\prime},[1.500000 \mathrm{e}+11,0.0,7600.0]\right)$

reaction('CH2CO + $\mathrm{CH} 3<=>\mathrm{AC} 3 \mathrm{H} 4+\mathrm{OH}$ ', [1.500000e+11, 0.0, 32300.0])

reaction('HCCO $\left.+\mathrm{OH}<=>\mathrm{CO}+\mathrm{H}+\mathrm{HCO}^{\prime},[1.000000 \mathrm{e}+13,0.0,0.0]\right)$

reaction $\left(' \mathrm{C} 3 \mathrm{H} 3+\mathrm{HCCO}<=>\mathrm{C} 4 \mathrm{H} 4+\mathrm{CO}^{\prime},[1.000000 \mathrm{e}+12,0.0,0.0]\right)$

reaction $\left(' \mathrm{CH} 2 \mathrm{CHCH} 2+\mathrm{HCCO}<=>\mathrm{C} 4 \mathrm{H} 6+\mathrm{CO}^{\prime},[1.000000 \mathrm{e}+12,0.0,0.0]\right)$

reaction('CYC5H5 + O => HCCO + $\mathrm{PC} 3 \mathrm{H} 4$ ', [5.000000e+12, 0.0, 0.0])

three_body_reaction('C2H5 + OH $\left.+\mathrm{M} \Leftrightarrow=>\mathrm{C} 2 \mathrm{H} 5 \mathrm{OH}+\mathrm{M}^{\prime},[1.000000 \mathrm{e}+16,0.0,-10000.0]\right)$

falloff_reaction('C2H5OH $(+\mathrm{M})<=>\mathrm{C} 2 \mathrm{H} 4+\mathrm{H} 2 \mathrm{O}(+\mathrm{M})$ ',

$\mathrm{kf}=[8.000000 \mathrm{e}+13,0.0,67900.0]$,

$\mathrm{kf0}=[1.000000 \mathrm{e}+17,0.0,53918.0]$,

efficiencies='AR:0.7 CH4:2.0 CO:1.5 CO2:2.0 H2:2.0 H2O:6.0 HE:0.7 ',

falloff $=\operatorname{Troe}(\mathrm{A}=0.5, \mathrm{~T} 3=1 \mathrm{e}-30, \mathrm{~T} 1=1.0000000000000002 \mathrm{e}+30, \mathrm{~T} 2=0.0))$

reaction $(' \mathrm{C} 2 \mathrm{H} 5 \mathrm{OH}+\mathrm{HO} 2=>0.33 \mathrm{C} 2 \mathrm{H} 4 \mathrm{OH}+0.67 \mathrm{CH} 3 \mathrm{CHOH}+\mathrm{H} 2 \mathrm{O} 2$ ', [1.000000e+13, 0.0, 16000.0])

reaction $(' \mathrm{CH} 2 \mathrm{CHCH} 2+\mathrm{OH}=>0.5 \mathrm{C} 2 \mathrm{H} 4+0.5 \mathrm{C} 3 \mathrm{H} 6 \mathrm{O}+0.5 \mathrm{CH} 2 \mathrm{O}$ ', [3.000000e+12, 0.0, 0.0])

reaction $(' \mathrm{C} 2 \mathrm{H} 3 \mathrm{CHO}+\mathrm{OH}=>\mathrm{C} 2 \mathrm{H} 2 \mathrm{O} 2+\mathrm{CH} 3$ ', [1.000000e+12, 0.0, 0.0])

reaction $\left(' \mathrm{C} 6 \mathrm{H} 10 \mathrm{O} 5=>\mathrm{C} 2 \mathrm{H} 3 \mathrm{CHO}+\mathrm{C} 2 \mathrm{H} 4 \mathrm{O} 2+\mathrm{HCO}+\mathrm{OH}^{\prime},[2.500000 \mathrm{e}+16,0.0,85000.0]\right)$

reaction $\left(' \mathrm{OH}+\mathrm{PC} 3 \mathrm{H} 4=>\mathrm{C} 2 \mathrm{H} 3 \mathrm{CHO}+\mathrm{H}^{\prime},[6.000000 \mathrm{e}+11,0.0,1000.0]\right)$

reaction $\left(' \mathrm{CH} 2 \mathrm{CHCH} 2+\mathrm{O} 2=>0.2 \mathrm{C} 2 \mathrm{H} 2+0.8 \mathrm{C} 2 \mathrm{H} 3 \mathrm{CHO}+0.2 \mathrm{CH} 2 \mathrm{O}+\mathrm{OH}^{\prime},[1.000000 \mathrm{e}+10,0.0,8000.0]\right)$

reaction $\left(' \mathrm{C} 6 \mathrm{H} 5 \mathrm{O}+\mathrm{OH}=>\mathrm{C} 4 \mathrm{H} 5+\mathrm{CO}+\mathrm{HCO}^{\prime},[5.000000 \mathrm{e}+12,0.0,0.0]\right)$

reaction $(' \mathrm{C} 6 \mathrm{H} 5 \mathrm{O}+\mathrm{OH}=>\mathrm{C} 6 \mathrm{H} 5+\mathrm{HO} 2$ ', [5.000000e+12, 0.0, 0.0])

reaction $\left(' \mathrm{C} 6 \mathrm{H} 5 \mathrm{O}+\mathrm{HO} 2=>2 \mathrm{C} 2 \mathrm{H} 2+\mathrm{CO}+\mathrm{HCO}+\mathrm{OH}^{\prime},[1.000000 \mathrm{e}+12,0.0,6000.0]\right)$

reaction $\left(' \mathrm{C} 6 \mathrm{H} 5 \mathrm{O}+\mathrm{OH}=>\mathrm{CYC} 5 \mathrm{H} 4 \mathrm{O}+\mathrm{H}+\mathrm{HCO}^{\prime},[3.000000 \mathrm{e}+12,0.0,0.0]\right)$

reaction $\left(' \mathrm{C} 7 \mathrm{H} 7+\mathrm{HO} 2=>\mathrm{C} 6 \mathrm{H} 5+\mathrm{CH} 2 \mathrm{O}+\mathrm{OH}^{\prime},[5.000000 \mathrm{e}+12,0.0,0.0]\right)$

reaction('C7H7 + O $\Leftrightarrow=>\mathrm{C} 6 \mathrm{H} 5+\mathrm{CH} 2 \mathrm{O}$ ', [8.000000e+13, 0.0, 0.0])

reaction $(' \mathrm{C} 7 \mathrm{H} 7=>\mathrm{C} 3 \mathrm{H} 3+\mathrm{C} 4 \mathrm{H} 4$ ', $[1.000000 \mathrm{e}+14,0.0,83600.0])$

reaction('C3H3 + C4H4 => C7H7', [2.000000e+13, 0.0, 16000.0])

reaction('H + RCRESOLO $<=>$ CRESOL', $[1.500000 \mathrm{e}+14,0.0,0.0])$

reaction('H + RCRESOLC $<=>$ CRESOL', [1.500000e+14, 0.0, 0.0])

reaction ('RCRESOLO $=>\mathrm{C} 6 \mathrm{H6}+\mathrm{CO}+\mathrm{H}$ ', [2.500000e+11, 0.0, 44000.0])

reaction $(' \mathrm{RCRESOLO}=>0.5 \mathrm{C} 2 \mathrm{H} 4+\mathrm{CO}+\mathrm{CYC} 5 \mathrm{H} 5$ ', [2.500000e+11, 0.0, 44000.0])

reaction $\left(' \mathrm{CH} 3 \mathrm{COOH}=>\mathrm{CH} 3+\mathrm{CO}+\mathrm{OH}^{\prime},[7.500000 \mathrm{e}+15,0.0,91600.0]\right)$ 
reaction(' $\left.\mathrm{CH} 3 \mathrm{COOH}=>\mathrm{CH} 3+\mathrm{CO} 2+\mathrm{H}^{\prime},[2.500000 \mathrm{e}+14,0.0,88000.0]\right)$

reaction $\left(' \mathrm{CH} 2 \mathrm{CO}+\mathrm{HO} 2=>\mathrm{H} 2 \mathrm{O} 2+\mathrm{HCCO}^{\prime},[1.616000 \mathrm{e}+05,2.0,10613.33]\right)$

reaction $(' \mathrm{C} 2 \mathrm{H} 2+\mathrm{CH} 3=>\mathrm{C} 2 \mathrm{H}+\mathrm{CH} 4$ ', [7.806000e+04, 2.0, 11601.78])

reaction $(' \mathrm{C} 2 \mathrm{H} 4+\mathrm{HO} 2=>\mathrm{C} 2 \mathrm{H} 3+\mathrm{H} 2 \mathrm{O} 2$ ', [4.310000e+05, 2.0, 20242.54])

reaction $(' \mathrm{C} 2 \mathrm{H} 6+\mathrm{HO} 2=>\mathrm{C} 2 \mathrm{H} 5+\mathrm{H} 2 \mathrm{O} 2$ ', [4.849000e+05, 2.0, 14841.16])

reaction(' $\mathrm{C} 2 \mathrm{H} 6+\mathrm{CH} 3=>\mathrm{C} 2 \mathrm{H} 5+\mathrm{CH} 4$ ', [3.513000e+05, 2.0, 7621.99])

reaction $(' \mathrm{C} 3 \mathrm{H} 6+\mathrm{CH} 3=>\mathrm{CH} 2 \mathrm{CHCH} 2+\mathrm{CH} 4$ ', [1.171000e+05, 2.0, 5617.33])

reaction('C3H6 + O $\left.=>\mathrm{CHCHCH} 3+\mathrm{OH}^{\prime},[5.921000 \mathrm{e}+06,2.0,8519.89]\right)$

reaction ('C3H6 + CH3 $\Rightarrow>\mathrm{CH} 4+\mathrm{CHCHCH} 3$ ', [1.708000e+05, 2.0, 11075.95])

reaction('C4H4 + O $\Rightarrow$ C $\left.4 \mathrm{H} 3+\mathrm{OH}^{\prime},[1.895000 \mathrm{e}+07,2.0,8519.89]\right)$

reaction(' $\mathrm{C} 4 \mathrm{H} 4+\mathrm{CH} 3=>\mathrm{C} 4 \mathrm{H} 3+\mathrm{CH} 4$ ', [5.464000e+05, 2.0, 11163.39])

reaction $(' \mathrm{H}+\mathrm{NC} 4 \mathrm{H} 8=>\mathrm{CH} 2 \mathrm{C} 3 \mathrm{H} 5+\mathrm{H} 2$ ', [7.220000e+06, 2.0, 6525.57])

reaction('NC4H8 $+\mathrm{OH}=>\mathrm{CH} 2 \mathrm{C} 3 \mathrm{H} 5+\mathrm{H} 2 \mathrm{O}$ ', [1.198000e+06, 2.0, -474.43])

reaction('NC4H8 + O => CH2C3H5 + OH', [4.060000e+06, 2.0, 5025.57])

reaction $\left(' \mathrm{H} 2 \mathrm{O}+\mathrm{HO} 2=>\mathrm{H} 2 \mathrm{O} 2+\mathrm{OH}^{\prime},[5.388000 \mathrm{e}+05,2.0,28780.05]\right)$

reaction('C7H7 + $\mathrm{H} 2 \mathrm{O}=>\mathrm{C} 7 \mathrm{H} 8+\mathrm{OH}$ ', [2.700000e+05, 2.0, 30572.64])

reaction(' $\mathrm{CH} 3 \mathrm{OH}+\mathrm{O} \Rightarrow>\mathrm{CH} 3 \mathrm{O}+\mathrm{OH}$ ', [6.767000e+05, 2.0, 4151.99])

reaction $(' \mathrm{CH} 3+\mathrm{CH} 3 \mathrm{OH}=>\mathrm{CH} 3 \mathrm{O}+\mathrm{CH} 4$ ', [1.952000e+04, 2.0, 5923.34])

reaction(' $\left.\mathrm{CH} 3 \mathrm{CHO}+\mathrm{O}=>\mathrm{CH} 3 \mathrm{CO}+\mathrm{OH}^{\prime},[8.120000 \mathrm{e}+06,2.0,1094.46]\right)$

reaction $(' \mathrm{CH} 3+\mathrm{CH} 3 \mathrm{CHO}=>\mathrm{CH} 3 \mathrm{CO}+\mathrm{CH} 4$ ', [2.342000e+05, 2.0, 3916.44])

reaction(' $\mathrm{CH} 3 \mathrm{CHO}+\mathrm{H}=>\mathrm{CH} 2 \mathrm{CHO}+\mathrm{H} 2$ ', [7.220000e+06, 2.0, 6675.07])

reaction(' $\mathrm{CH} 3 \mathrm{CHO}+\mathrm{OH}=>\mathrm{CH} 2 \mathrm{CHO}+\mathrm{H} 2 \mathrm{O}$ ', [1.198000e+06, 2.0, 610.83])

reaction ('CH3CHO $+\mathrm{O}=>\mathrm{CH} 2 \mathrm{CHO}+\mathrm{OH}^{\prime}$, [4.060000e+06, 2.0, 5025.57])

reaction $\left(' \mathrm{O}+\mathrm{PC} 3 \mathrm{H} 4=>\mathrm{C} 3 \mathrm{H} 3+\mathrm{OH}^{\prime},[8.120000 \mathrm{e}+07,2.0,8781.96]\right)$

reaction('MCPTD + OH => C6H6 + H + H2O', [3.994000e+06, 2.0, -3152.53])

reaction $(' \mathrm{C} 2 \mathrm{H} 5 \mathrm{OH}+\mathrm{OH}=>0.67 \mathrm{CH} 2 \mathrm{O}+0.67 \mathrm{CH} 3+0.33 \mathrm{CH} 3 \mathrm{CHO}+0.33 \mathrm{H}+\mathrm{H} 2 \mathrm{O}$ ', [3.994000e+05, 2.0, -474.43])

reaction $(' \mathrm{C} 2 \mathrm{H} 5 \mathrm{OH}+\mathrm{H}=>\mathrm{C} 2 \mathrm{H} 4 \mathrm{OH}+\mathrm{H} 2$ ', [7.220000e+06, 2.0, 6525.57])

reaction(' $\left.\mathrm{C} 2 \mathrm{H} 5 \mathrm{OH}+\mathrm{O}=>\mathrm{C} 2 \mathrm{H} 4 \mathrm{OH}+\mathrm{OH}^{\prime},[4.060000 \mathrm{e}+06,2.0,5025.57]\right)$

reaction $\left(' \mathrm{C} 2 \mathrm{H} 4 \mathrm{O} 2+\mathrm{O}=>\mathrm{C} 2 \mathrm{H} 2 \mathrm{O} 2+\mathrm{H}+\mathrm{OH}^{\prime},[1.353000 \mathrm{e}+06,2.0,2579.54]\right)$

reaction $(' \mathrm{C} 2 \mathrm{H} 4 \mathrm{O} 2+\mathrm{HO} 2=>\mathrm{C} 2 \mathrm{H} 2 \mathrm{O} 2+\mathrm{H}+\mathrm{H} 2 \mathrm{O} 2$ ', [5.388000e+04, 2.0, 11887.73])

reaction $\left(' \mathrm{C} 2 \mathrm{H} 4 \mathrm{O} 2+\mathrm{CH} 3=>\mathrm{C} 2 \mathrm{H} 2 \mathrm{O} 2+\mathrm{CH} 4+\mathrm{H}^{\prime},[3.903000 \mathrm{e}+04,2.0,4871.29]\right)$

reaction $\left(' \mathrm{C} 3 \mathrm{H} 4 \mathrm{O} 3+\mathrm{CH} 3=>\mathrm{C} 2 \mathrm{H} 2 \mathrm{O} 2+\mathrm{CH} 4+\mathrm{CO}+\mathrm{H}^{\prime},[3.122000 \mathrm{e}+05,2.0,3259.77]\right)$

reaction $(' \mathrm{C} 6 \mathrm{H} 6 \mathrm{O} 3+\mathrm{HO} 2=>\mathrm{C} 5 \mathrm{H} 4 \mathrm{O} 2+\mathrm{CO}+\mathrm{H}+\mathrm{H} 2 \mathrm{O} 2$ ', [2.155000e+05, 2.0, 9982.61])

reaction $(' \mathrm{C} 5 \mathrm{H} 4 \mathrm{O} 2+\mathrm{HO} 2=>\mathrm{C} 3 \mathrm{H} 3+2 \mathrm{CO}+\mathrm{H} 2 \mathrm{O} 2 ',[2.155000 \mathrm{e}+05,2.0,9982.61])$

reaction $(' \mathrm{C} 5 \mathrm{H} 4 \mathrm{O} 2+\mathrm{HO} 2=>\mathrm{C} 4 \mathrm{H} 3 \mathrm{O}+\mathrm{CO}+\mathrm{H} 2 \mathrm{O} 2$ ', [2.155000e+05, 2.0, 9982.61])

reaction('C5H4O2 $\left.+\mathrm{CH} 3=>\mathrm{C} 4 \mathrm{H} 3 \mathrm{O}+\mathrm{CH} 4+\mathrm{CO}^{\prime},[1.561000 \mathrm{e}+05,2.0,3259.77]\right)$

reaction('C6H10O5 $\left.+\mathrm{O}=>\mathrm{C} 3 \mathrm{H} 4 \mathrm{O} 3+\mathrm{CH} 2 \mathrm{CHO}+\mathrm{CH} 2 \mathrm{O}+\mathrm{OH}^{\prime},[5.413000 \mathrm{e}+06,2.0,2579.54]\right)$

reaction $(' \mathrm{C} 6 \mathrm{H} 10 \mathrm{O} 5+\mathrm{HO} 2=>\mathrm{C} 3 \mathrm{H} 4 \mathrm{O} 3+\mathrm{CH} 2 \mathrm{CHO}+\mathrm{CH} 2 \mathrm{O}+\mathrm{H} 2 \mathrm{O} 2 ',[2.155000 \mathrm{e}+05,2.0,11887.73])$

reaction('C6H10O5 $+\mathrm{CH} 3=>\mathrm{C} 3 \mathrm{H} 4 \mathrm{O} 3+\mathrm{CH} 2 \mathrm{CHO}+\mathrm{CH} 2 \mathrm{O}+\mathrm{CH} 4$ ', [1.561000e+05, 2.0, 4871.29])

reaction ('C5H8O4 $+\mathrm{O}=>\mathrm{C} 2 \mathrm{H} 4 \mathrm{O} 2+\mathrm{CH} 2 \mathrm{CHO}+\mathrm{CO}+\mathrm{OH}^{\prime}$, [2.707000e+06, 2.0, 2579.54])

reaction $\left(' \mathrm{C} 5 \mathrm{H} 8 \mathrm{O} 4+\mathrm{CH} 3=>\mathrm{C} 2 \mathrm{H} 4 \mathrm{O} 2+\mathrm{CH} 2 \mathrm{CHO}+\mathrm{CH} 4+\mathrm{CO}^{\prime},[7.806000 \mathrm{e}+04,2.0,4871.29]\right)$

reaction $(' \mathrm{C} 5 \mathrm{H} 8 \mathrm{O} 4+\mathrm{CH} 3=>\mathrm{C} 2 \mathrm{H} 2 \mathrm{O} 2+0.5 \mathrm{C} 2 \mathrm{H} 3 \mathrm{CHO}+0.5 \mathrm{CH} 3 \mathrm{CHO}+\mathrm{CH} 4+0.5 \mathrm{HCO}+0.5 \mathrm{OH}$, [7.806000e+04, 2.0, 4871.29]) 
reaction(' $\mathrm{C} 8 \mathrm{H} 10 \mathrm{O} 3+\mathrm{OH}=>\mathrm{C} 2 \mathrm{H} 2+0.5 \mathrm{C} 4 \mathrm{H} 4+\mathrm{CH} 2 \mathrm{CO}+\mathrm{CH} 3 \mathrm{O}+\mathrm{CO}+\mathrm{H} 2 \mathrm{O}$ ', [1.997000e+06, 2.0, -474.43])

reaction (' $\left.\mathrm{C} 2 \mathrm{H} 3 \mathrm{CHO}+\mathrm{O}=>0.1 \mathrm{C} 2 \mathrm{H} 2+0.9 \mathrm{C} 2 \mathrm{H} 3+0.9 \mathrm{CO}+0.1 \mathrm{HCO}+\mathrm{OH}^{\prime},[8.120000 \mathrm{e}+06,2.0,1094.46]\right)$

reaction $\left(' \mathrm{C} 2 \mathrm{H} 3 \mathrm{CHO}+\mathrm{HO} 2=>0.1 \mathrm{C} 2 \mathrm{H} 2+0.9 \mathrm{C} 2 \mathrm{H} 3+0.9 \mathrm{CO}+\mathrm{H} 2 \mathrm{O} 2+0.1 \mathrm{HCO}^{\prime},[3.233000 \mathrm{e}+05,2.0,9982.61]\right)$

reaction(' $\mathrm{C} 3 \mathrm{H} 6 \mathrm{O}+\mathrm{H}=>\mathrm{C} 2 \mathrm{H} 3 \mathrm{CHO}+\mathrm{H}+\mathrm{H} 2$ ', [3.610000e+06, 2.0, 3950.57])

reaction $(' \mathrm{C} 6 \mathrm{H} 5 \mathrm{OH}+\mathrm{CH} 3=>\mathrm{C} 6 \mathrm{H} 5 \mathrm{O}+\mathrm{CH} 4 ',[1.952000 \mathrm{e}+05,2.0,8062.59])$

reaction('C7H8 + H => C7H7 + H2', [7.220000e+06, 2.0, 4189.23])

reaction('CRESOL + OH $=>\mathrm{H} 2 \mathrm{O}+0.75$ RCRESOLC + 0.25 RCRESOLO', [3.195000e+06, 2.0, -2259.83])

reaction $(' \mathrm{CH} 3 \mathrm{COOH}+\mathrm{O}=>\mathrm{CH} 2 \mathrm{CO}+2 \mathrm{OH}$ ', [4.060000e+06, 2.0, 5025.57])

reaction $\left(' \mathrm{CH} 3 \mathrm{COOH}+\mathrm{HO} 2=>\mathrm{CH} 2 \mathrm{CO}+\mathrm{H} 2 \mathrm{O} 2+\mathrm{OH}^{\prime},[1.616000 \mathrm{e}+05,2.0,15025.57]\right)$

reaction $(' \mathrm{CH} 3+\mathrm{CH} 3 \mathrm{COOH}=>\mathrm{CH} 2 \mathrm{CO}+\mathrm{CH} 4+\mathrm{OH}$ ', [1.171000e+05, 2.0, 7525.57])

reaction $\left(' \mathrm{CH} 3 \mathrm{COOH}+\mathrm{O}=>\mathrm{CH} 3+\mathrm{CO} 2+\mathrm{OH}^{\prime},[1.353000 \mathrm{e}+06,2.0,5025.57]\right)$

reaction $(' \mathrm{C} 3 \mathrm{H} 6 \mathrm{O} 2+\mathrm{H}=>\mathrm{C} 2 \mathrm{H} 4 \mathrm{OH}+\mathrm{CO}+\mathrm{H} 2$ ', [6.739000e+06, 2.0, 2387.18]) 